\title{
MOMENT MEASURES OF HEAVY-TAILED RENEWAL POINT PROCESSES: ASYMPTOTICS AND APPLICATIONS
}

\author{
Clément Dombry ${ }^{1}$ And Ingemar $\mathrm{KAJ}^{2}$
}

\begin{abstract}
We study higher-order moment measures of heavy-tailed renewal models, including a renewal point process with heavy-tailed inter-renewal distribution and its continuous analog, the occupation measure of a heavy-tailed Lévy subordinator. Our results reveal that the asymptotic structure of such moment measures are given by explicit power-law density functions. The same power-law densities appear naturally as cumulant measures of certain Poisson and Gaussian stochastic integrals. This correspondence provides new and extended results regarding the asymptotic fluctuations of heavy-tailed sources under aggregation, and clarifies existing links between renewal models and fractional random processes.
\end{abstract}

Mathematics Subject Classification. 60K05, 60G22, 60F05.

Received February 21, 2012.

\section{INTRODUCTION AND MAIN RESULTS}

The topic of study in this work is heavy-tailed renewal models, starting from a renewal point process on the line with an inter-renewal time distribution which is heavy-tailed at infinity. The occupation measure of a Lévy subordinator with heavy-tailed Lévy measure provides a continuous analog. Our analysis shows that the discrete and the continuous models are closely related with respect to the structure of their higher-order moment measures. We show that the moment measures in both cases have the same asymptotic structure and that the limit measures are given by specific density functions with a power-law behavior governed by the inter-renewal tail index. As customary, our approach to the discrete renewal model begins with properties of factorial measures.

The power-law density functions which are recognized to determine the asymptotic behavior of moments in the renewal models also appear naturally as cumulant measures of certain Poisson and Gaussian stochastic integrals. This correspondence helps us to unify and extend results on asymptotic fluctuations of heavy-tailed sources under aggregation, and to clarify the role in this context of fractional Poisson motion $[2,5]$ and of fractional Brownian motion as rescaling limit processes.

Hence we believe that the present paper sheds some new light on the rich literature on traffic models and aggregation of heavy-tailed sources. Popular models include the infinite source Poisson model [10,13], the

\footnotetext{
Keywords and phrases. Heavy-tailed renewal process, moment measures, fractional Brownian motion, fractional Poisson motion.

1 Laboratoire LMA, Université de Poitiers, Téléport 2, BP 30179, 86962 Futuroscope-Chasseneuil Cedex, France.

clement.dombry@math.univ-poitiers.fr

2 Department of Mathematics, Uppsala University, Box 480 SE 75106 Uppsala, Sweden. ikaj@math.uu.se
} 
aggregation of ON/OFF sources [5,13,19], renewal processes [7] or renewal/reward processes $[12,14,15]$. The interested reader should also refer to the monography on heavy-tailed phenomena [17].

We begin this first section of the paper by introducing a renewal point process on the positive half line with heavy-tailed inter-renewal distribution at infinity. Then we introduce the relevant families of power-law density functions, defined on $[0, \infty)^{k}$ for each $k \geq 1$, and recognize the role of these families of functions within two separate frameworks. Firstly they arise as asymptotic limits of the higher-order moment measures for the renewal model. Secondly, the same families of power-law measures express the cumulants for a class of Poisson stochastic integrals with power-law intensity measure. Closely related are Gaussian stochastic integrals defined by a power-law control measure. Based on these links we establish a limit theorem for renewal point processes under aggregation and scaling, and show that the asymptotic fluctuations are given by Poisson or Gaussian stochastic integrals. These are natural extensions of fractional Poisson motion and fractional Brownian motion, which explains and extends earlier results on scaling limits of heavy-tailed renewal counting processes.

All results relating to higher order moment measures of the renewal model have been collected in Section 2 . We investigate systematically the asymptotic behavior of factorial moment measures, moment measures and centered moment measures, leading up to identifying the limiting centered moments in terms of power-law density functions. In Section 3 we apply these findings in order to present the proof of our main result Theorem 1.4, which gives the fluctuation limits for renewal systems under simultaneous aggregation and time scaling.

In Section 4 these results are extended to a continuous version of the discrete renewal model based on the occupation measure of a heavy-tailed Lévy subordinator. We establish that the same power-law density measures as for the discrete renewal model provide the higher-order moment asymptotics and the same Poisson and Gaussian integrals yield the scaling limits under aggregation.

\subsection{A discrete renewal model}

Consider the renewal sequence $\left(S_{n}\right)_{n \geq 0}$ generated by a sequence of independent non-negative random variables $\left(X_{k}\right)_{k \geq 1}$, i.e. $S_{n}=S_{0}+\sum_{i=1}^{n} X_{i}$ for $n \geq 1$. The inter-renewal times $\left(X_{k}\right)_{k \geq 1}$ are identically distributed with probability measure $F$ and independent of $S_{0}$. We write $x \in \mathbb{R}^{+} \mapsto F([0, x])$ for the distribution function and assume that the inter-renewal distribution has finite mean $\mu=\int_{0}^{\infty} F([x, \infty)) \mathrm{d} x>0$. The initial distribution, i.e. the distribution of $S_{0}$, is denoted by $\pi$. We will use the notation $\mathbb{P}_{\pi}, \mathbb{E}_{\pi}$ for the probability measure and expectation of the renewal model with initial distribution $\pi$. Two main cases appear naturally: the pure renewal case where $S_{0} \equiv 0$, i.e. $\pi=\delta_{0}$, and the stationary renewal case where $S_{0}$ follows the equilibrium distribution

$$
\pi_{\mathrm{eq}}(x)=\frac{1}{\mu} \int_{0}^{x} F([s, \infty)) \mathrm{d} s, \quad x \geq 0 .
$$

Probability and expectation are denoted by $\mathbb{P}_{0}$ and $\mathbb{E}_{0}$ in the pure case $\pi=\delta_{0}$ and by $\mathbb{P}_{\text {eq }}$ and $\mathbb{E}_{\text {eq }}$ in the stationary case $\pi=\pi_{\text {eq }}$.

Recall that a function $\ell:(0, \infty) \rightarrow(0, \infty)$ is said to be slowly varying at infinity if for all $t>0, \ell(t x) / \ell(x) \rightarrow 1$, $x \rightarrow \infty$. Our basic assumption is that the inter-renewal distribution $F$, in addition to finite expected value $\mu$, has a regularly varying tail with exponent $1+\beta, \beta \in(0,1)$, i.e. there exists a slowly varying function $\ell$ such that

$$
F([x, \infty)) \sim x^{-(1+\beta)} \ell(x) \text { as } x \rightarrow \infty .
$$

Thus, $X_{k}, k \geq 1$, have infinite variance. To study the renewal model $\left(S_{n}\right)$ we will use general ideas of renewal point processes and moment measures, see [4] for a full account of such methods.

The renewal point process $\xi=\sum_{n \geq 0} \delta_{S_{n}}$ on $[0,+\infty)$ is locally finite under $\mathbb{P}_{\pi}$. For $k \geq 1$, the $k$-fold tensorial product of $\xi$ with itself is the random point measure on $[0,+\infty)^{k}$ given by

$$
\xi^{\otimes k}=\sum_{n_{1}, \ldots, n_{k} \geq 0} \delta_{\left(S_{n_{1}}, \ldots, S_{n_{k}}\right)} .
$$


For $k \geq 1$, we consider $[0,+\infty)^{k}$ endowed with the Borel $\sigma$-algebra $\mathcal{B}_{k}$. The $k$ th moment measure $M_{k}^{\pi}$ of $\xi$ is the measure on $[0,+\infty)^{k}$ defined by

$$
M_{k}^{\pi}(A)=\mathbb{E}_{\pi}\left[\xi^{\otimes k}(A)\right], \quad A \in \mathcal{B}_{k} .
$$

We also define a centered version of the $k$ th moment measure by

$$
\widetilde{M}_{k}^{\pi}(A)=\mathbb{E}_{\pi}\left[\left(\xi-\lambda_{1} / \mu\right)^{\otimes k}(A)\right], \quad A \in \mathcal{B}_{k} \text { bounded, }
$$

where $\lambda_{1}$ is Lebesgue measure on the real line. In particular, for $k=1$,

$$
M_{1}^{0}(A)=\mathbb{E}_{0}[\xi(A)], \quad M_{1}^{\mathrm{eq}}(A)=\frac{1}{\mu} \lambda_{1}(A), \quad \widetilde{M}_{1}^{0}(A)=\mathbb{E}_{0}[\xi(A)]-\frac{1}{\mu} \lambda_{1}(A), \quad \widetilde{M}_{1}^{\mathrm{eq}}(A)=0 .
$$

For the special case of cylinder sets in $[0,+\infty)^{k}$ we have

$$
\xi^{\otimes k}\left(\bigotimes_{i=1}^{k} A_{i}\right)=\prod_{i=1}^{k} \xi\left(A_{i}\right), \quad A_{1}, \ldots A_{k} \in \mathcal{B}_{1} .
$$

For closed intervals $A_{i}=\left[0, x_{i}\right]$, this is $\xi^{\otimes k}\left(\bigotimes_{i=1}^{k}\left[0, x_{i}\right]\right)=\prod_{i=1}^{k} \xi\left(\left[0, x_{i}\right]\right)$, which evaluates tensorial products in terms of the renewal counting process

$$
\xi([0, x])=\operatorname{Card}\left\{n \geq 0 ; S_{n} \leq x\right\}, \quad x \geq 0 .
$$

Similarly, our detailed analysis of higher order moment measures will reveal representations in terms of first order moments, that is the familiar renewal measures

$$
U=\sum_{n=1}^{\infty} F^{* n}, \quad U+\delta_{0}=\sum_{n=0}^{\infty} F^{* n}
$$

where $F^{* n}$ is the $n$-fold convolution of $F$ and $F^{* 0}=\delta_{0}$ is Dirac mass at 0 . Here, $x \mapsto U([0, x])$ is the associated renewal function.

\subsection{A collection of measures}

To prepare for our analysis of renewal moment measures we introduce four families of measures defined on $[0,+\infty)^{k}, k \geq 1$. They are parameterized by the tail index $\beta, 0<\beta<1$ introduced in (1.2) and defined by their densities with respect to the Lebesgue measure $\lambda_{k}, \lambda_{k}(\mathrm{~d} x)=\mathrm{d} x, x=\left(x_{1}, \ldots, x_{k}\right)$. The measures will be denoted $P_{k}^{\pi}$ and $\widetilde{P}_{k}^{\pi}, k \geq 1$, with the tilde-mark indicating a centered measure and the upper index $\pi$ an initial distribution. Whenever it is suitable to distinguish the two choices of initial distributions we use upper index 0 for the case $\pi=\delta_{0}$ and eq for the equilibrium distribution $\pi=\pi_{\mathrm{eq}}$ in (1.1). For $k=1$, define

$$
\frac{\mathrm{d} P_{1}^{0}}{\mathrm{~d} \lambda_{1}}(x)=|x|^{-\beta}, \quad \frac{\mathrm{d} \widetilde{P}_{1}^{0}}{\mathrm{~d} \lambda_{1}}(x)=-|x|^{-\beta} \quad \text { and } \quad \frac{\mathrm{d} P_{1}^{\text {eq }}}{\mathrm{d} \lambda_{1}}(x)=\frac{\mathrm{d} \widetilde{P}_{1}^{\text {eq }}}{\mathrm{d} \lambda_{1}}(x)=0 .
$$

We use here the convention $0^{-\beta}=\infty$ so that the densities may be infinite on a set of zero Lebesgue measure. For $k \geq 2$, let $x_{(1)} \leq \ldots \leq x_{(k)}$ be the order statistic of the vector $x=\left(x_{1}, \ldots, x_{k}\right) \in[0,+\infty)^{k}$ and put

$$
\frac{\mathrm{d} P_{k}^{0}}{\mathrm{~d} \lambda_{k}}(x)=\left|x_{(1)}\right|^{-\beta}+\sum_{i=2}^{k}\left|x_{(i)}-x_{(i-1)}\right|^{-\beta}, \quad \frac{\mathrm{d} \widetilde{P}_{k}^{0}}{\mathrm{~d} \lambda_{k}}(x)=\left|x_{(k)}-x_{(1)}\right|^{-\beta}-\left|x_{(k)}\right|^{-\beta}
$$


and

$$
\frac{\mathrm{dP}}{\mathrm{d} \lambda_{k}}(x)=\sum_{i=2}^{\mathrm{eq}}\left|x_{(i)}-x_{(i-1)}\right|^{-\beta}, \quad \frac{\mathrm{d} \widetilde{\mathrm{P}}_{k}^{\mathrm{eq}}}{\mathrm{d} \lambda_{k}}(x)=\left|x_{(k)}-x_{(1)}\right|^{-\beta} .
$$

These measures will play a key role in this work as they provide non-obvious links between the renewal models on one hand and Poisson integrals on the other. It is worth noting that they enjoy interesting scaling properties: for all $a>0$ and $A \in \mathcal{B}_{k}$,

$$
P_{k}^{\pi}(a A)=a^{k-\beta} P_{k}^{\pi}(A), \quad \widetilde{P}_{k}^{\pi}(a A)=a^{k-\beta} \widetilde{P}_{k}^{\pi}(A),
$$

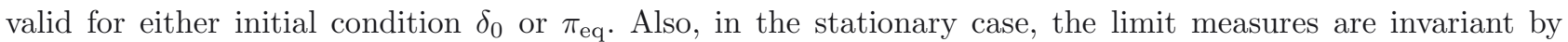
translation: for all $h \geq 0$ and $A \in \mathcal{B}_{k}$

$$
P_{k}^{\mathrm{eq}}(A+h)=P_{k}^{\mathrm{eq}}(A), \quad \widetilde{P}_{k}^{\mathrm{eq}}(A+h)=\widetilde{P}_{k}^{\mathrm{eq}}(A) .
$$

In the pure case, for all bounded $A \in \mathcal{B}_{k}$,

$$
\lim _{h \rightarrow+\infty} P_{k}^{0}(A+h)=P_{k}^{\mathrm{eq}}(A), \quad \lim _{h \rightarrow+\infty} \widetilde{P}_{k}^{0}(A+h)=\widetilde{P}_{k}^{\mathrm{eq}}(A) .
$$

\subsection{Scaling limits for moment measures}

We are interested in the asymptotic behavior of the moment measures under rescaling of the space $[0,+\infty)$. For $a>0$ and $k \geq 1$, let $s_{a}=s_{a}^{k}$ denote the component wise defined scaling map $x \mapsto x / a$ from $[0,+\infty)^{k}$ onto itself. We define the rescaled point process $\xi s_{a}^{-1}$ by

$$
\xi s_{a}^{-1}(A)=\xi(a A), \quad A \in \mathcal{B}_{k},
$$

and notice $\xi s_{a}^{-1}=\sum_{n \geq 0} \delta_{S_{n} / a}$. The $k$ th moment measure $M_{k}^{\pi} s_{a}^{-1}$ and the $k$ th centered moment measure $\widetilde{M}_{k}^{\pi} s_{a}^{-1}$ of $\xi s_{a}^{-1}$, are given by

$$
M_{k}^{\pi} s_{a}^{-1}(A)=M_{k}^{\pi}(a A), \quad \widetilde{M}_{k}^{\pi} s_{a}^{-1}(A)=\widetilde{M}_{k}^{\pi}(a A), \quad A \in \mathcal{B}_{k} .
$$

The asymptotic behavior of the rescaled moment measures as $a \rightarrow \infty$ will be given in terms of weak convergence as follows. If $m$ is a signed Radon measure and $f$ a bounded and compactly supported function both defined on $[0, \infty)^{+}$we write $m[f]=\int f d m$, for the corresponding integral. Let

$$
\mathcal{F}_{k}=\left\{f:[0,+\infty)^{k} \rightarrow \mathbb{R} ; \text { compact support }, \frac{\partial^{k} f}{\partial x_{1} \ldots \partial x_{k}} \text { exists and is continuous }\right\}, \quad k \geq 1 .
$$

The technical reason for this choice of test functions is the use of an integration by parts formula (see Lem. 2.1 below) and the fact that $f^{\otimes k} \in \mathcal{F}_{k}$ when $f \in \mathcal{F}_{1}$. If $\left(m_{n}\right)_{n \geq 1}$ and $m$ are signed Radon measures on $[0,+\infty)^{k}$, we say that $\left(m_{n}\right)_{n \geq 1} \mathcal{F}_{k}$-converges to $m$, denoted $m_{n} \stackrel{\mathcal{F}_{k}}{\longrightarrow} m$, if and only if

$$
m_{n}[f] \rightarrow m[f], \quad n \rightarrow \infty, \quad f \in \mathcal{F}_{k} .
$$

Our main result for the discrete renewal model is the following limit theorem, which identifies the asymptotic form of $k$-moment measures expressed in terms of the power law measures $P_{k}^{\pi}$ and $\widetilde{P}_{k}^{\pi}$.

Theorem 1.1. Suppose that assumption (1.2) is satisfied. For $\pi=\delta_{0}$ or $\pi=\pi_{\mathrm{eq}}$ and $k \geq 1$,

$$
\frac{M_{k}^{\pi} s_{a}^{-1}-(a / \mu)^{k} \lambda_{k}}{a^{k-\beta} \ell(a)} \stackrel{\mathcal{F}_{k}}{\longrightarrow} \frac{1}{\beta \mu^{k+1}} P_{k}^{\pi} \quad \text { and } \quad \frac{\widetilde{M}_{k}^{\pi} s_{a}^{-1}}{a^{k-\beta} \ell(a)} \stackrel{\mathcal{F}_{k}}{\longrightarrow} \frac{(-1)^{k}}{\beta \mu^{k+1}} \widetilde{P}_{k}^{\pi} \quad \text { as } a \rightarrow+\infty .
$$




\subsection{A class of Poisson and Gaussian integrals}

We relate the measures $\widetilde{P}_{k}^{\text {eq }}$ and $\widetilde{P}_{k}^{0}$ introduced in (1.4) to the cumulants of Poisson and Gaussian integrals. Recall that we have fixed a parameter $\beta \in(0,1)$ and let $N(\mathrm{~d} x, \mathrm{~d} u)$ be a Poisson random measure on $\mathbb{R} \times[0,+\infty)$ with intensity measure

$$
n(\mathrm{~d} x, \mathrm{~d} u)=\mathrm{d} x(\beta+1) u^{-\beta-2} \mathrm{~d} u
$$

and let $\tilde{N}(\mathrm{~d} x, \mathrm{~d} u)=N(\mathrm{~d} x, \mathrm{~d} u)-n(\mathrm{~d} x, \mathrm{~d} u)$ denote the corresponding compensated Poisson measure. Once again, we use the convention $0^{-\beta-2}=\infty$ and the intensity measure density may be infinite on a set with zero Lebesgue measure. Similarly, let $N_{+}(\mathrm{d} x, \mathrm{~d} u)$ be the Poisson random measure on $[0,+\infty) \times[0,+\infty)$ with intensity $n_{+}(\mathrm{d} x, \mathrm{~d} u)=1_{x \geq 0} n(\mathrm{~d} x, \mathrm{~d} u)$ and put $\widetilde{N}_{+}=N_{+}-n_{+}$.

It is convenient for our purpose to view each Poisson point $(x, u)$ as an interval $[x, x+u]$ on the real line and the Poisson measure $N(\mathrm{~d} x, \mathrm{~d} u)$ as a collection of overlapping sessions formed by these intervals. With this in mind we will restrict attention to integrands obtained as the weighted occupation times

$$
g(x, u)=\int_{[0,+\infty)} 1_{[x, x+u]}(y) f(y) \mathrm{d} y .
$$

For $f:[0,+\infty) \rightarrow \mathbb{R}$ and $k \geq 1$, we define the tensor product $f^{\otimes k}:[0,+\infty)^{k} \rightarrow \mathbb{R}$ by

$$
f^{\otimes k}\left(x_{1}, \ldots, x_{k}\right)=\prod_{i=1}^{k} f\left(x_{i}\right) .
$$

Proposition 1.2. For any measurable and bounded function $f:[0,+\infty) \rightarrow \mathbb{R}$ with compact support, the Poisson integrals

$$
\begin{aligned}
J_{\beta}^{\mathrm{eq}}[f] & =\int_{\mathbb{R} \times[0,+\infty)} \int_{[0,+\infty)} 1_{[x, x+u]}(y) f(y) \mathrm{d} y \tilde{N}(\mathrm{~d} x, \mathrm{~d} u), \\
J_{\beta}^{0}[f] & =\int_{[0,+\infty) \times[0,+\infty)} \int_{[0,+\infty)} 1_{[x, x+u]}(y) f(y) \mathrm{d} y \widetilde{N}_{+}(\mathrm{d} x, \mathrm{~d} u),
\end{aligned}
$$

are well defined and have finite cumulants of any order given by

$$
C_{k}\left(J_{\beta}^{\mathrm{eq}}[f]\right)=\frac{1}{\beta} \widetilde{P}_{k}^{\mathrm{eq}}\left[f^{\otimes k}\right], \quad C_{k}\left(J_{\beta}^{0}[f]\right)=\frac{1}{\beta} \widetilde{P}_{k}^{0}\left[f^{\otimes k}\right], \quad k \geq 2,
$$

and $C_{1}\left(J_{\beta}^{\mathrm{eq}}[f]\right)=C_{1}\left(J_{\beta}^{0}[f]\right)=0$. Furthermore, the distributions of $J_{\beta}^{\mathrm{eq}}[f]$ and $J_{\beta}^{0}[f]$ are uniquely determined by their sequence of cumulants.

We now define the Gaussian counterpart of these Poisson integrals, see e.g. [18] for background and details. Let $W(\mathrm{~d} x, \mathrm{~d} u)$ be a Gaussian random measure on $\mathbb{R} \times[0,+\infty)$ with control measure $n(\mathrm{~d} x, \mathrm{~d} u)$. Similarly, let $W_{+}(\mathrm{d} x, \mathrm{~d} u)$ be a Gaussian random measure on $[0,+\infty) \times[0,+\infty)$ with control measure $n_{+}(\mathrm{d} x, \mathrm{~d} u)$.

Proposition 1.3. For any measurable and bounded function $f:[0,+\infty) \rightarrow \mathbb{R}$ with compact support, the Gaussian integrals

$$
\begin{aligned}
G_{\beta}^{\mathrm{eq}}[f] & =\int_{\mathbb{R} \times[0,+\infty)} \int_{[0,+\infty)} 1_{[x, x+u]}(y) f(y) \mathrm{d} y W(\mathrm{~d} x, \mathrm{~d} u), \\
G_{\beta}^{0}[f] & =\int_{[0,+\infty) \times[0,+\infty)} \int_{[0,+\infty)} 1_{[x, x+u]}(y) f(y) \mathrm{d} y W_{+}(\mathrm{d} x, \mathrm{~d} u),
\end{aligned}
$$

are well defined and have finite cumulants of any order given by

$$
C_{2}\left(G_{\beta}^{\mathrm{eq}}[f]\right)=\frac{1}{\beta} \widetilde{P}_{2}^{\mathrm{eq}}\left[f^{\otimes 2}\right], \quad C_{2}\left(G_{\beta}^{0}[f]\right)=\frac{1}{\beta} \widetilde{P}_{2}^{0}\left[f^{\otimes 2}\right]
$$


and for $k=1$ or $k \geq 3$,

$$
C_{k}\left(G_{\beta}^{\mathrm{eq}}[f]\right)=0, \quad C_{k}\left(G_{\beta}^{0}[f]\right)=0 .
$$

\subsection{Scaling limits for superposition of sources}

Let $\xi^{i}, i \geq 1$ be i.i.d. copies of the random measure $\xi$. We will consider

$$
\widetilde{\xi}_{a, m}=\frac{1}{b(a, m)} \sum_{i=1}^{m}\left(\xi^{i} s_{a}^{-1}-M_{1}^{\pi} s_{a}^{-1}\right),
$$

obtained by aggregation of $m$ centered copies of $\xi$ under spatial scaling by $s_{a}$, with a suitable normalization sequence $b(a, m)$, and study the asymptotic behavior of $\widetilde{\xi}_{a, m}$ as $a \rightarrow \infty$ and $m \rightarrow \infty$. To deal with the simultaneous limit, we consider $a=a_{m} \rightarrow+\infty$ as $m \rightarrow+\infty$.

The two limit regimes of interest to us are known in some applications as regimes of intermediate connection rate and of fast connection rate, respectively, and are hence labeled in the following (ICR) and (FCR). The intermediate scaling condition is given by

$$
\frac{m \ell(a)}{a^{\beta}} \rightarrow \mu c^{\beta}, \quad 0<c<\infty,
$$

whereas the fast scaling condition entails

$$
\frac{m \ell(a)}{a^{\beta}} \rightarrow+\infty
$$

We consider the random fields $\left\{\widetilde{\xi}_{a, m}[f]: f \in \mathcal{F}_{1}\right\}$ and prove convergence of the finite dimensional distributions, which we denote by $\stackrel{f i d i}{\longrightarrow}$. For $f \in \mathcal{F}_{1}$, we define $f_{c}$ by $f_{c}(x)=c f(c x)$.

\section{Theorem 1.4.}

(1) Consider the rescaling assumption (ICR) with normalization $b(a, m)=a$.

In the pure case $\pi=\delta_{0}$,

$$
\widetilde{\xi}_{a, m}[f] \stackrel{f i d i}{\longrightarrow}-\frac{1}{\mu} J_{\beta}^{0}\left[f_{c}\right], \quad f \in \mathcal{F}_{1}
$$

in the stationary case $\pi=\pi_{\mathrm{eq}}$,

$$
\widetilde{\xi}_{a, m}[f] \stackrel{\text { fidi }}{\longrightarrow}-\frac{1}{\mu} J_{\beta}^{\mathrm{eq}}\left[f_{c}\right], \quad f \in \mathcal{F}_{1} .
$$

(2) Under the scaling assumption (FCR) with normalization $b(a, m)=\left(m a^{2-\beta} \ell(a)\right)^{1 / 2}$, in the pure case $\pi=\delta_{0}$

$$
\widetilde{\xi}_{a, m}[f] \stackrel{f i d i}{\longrightarrow} G_{\beta}^{0}[f], \quad f \in \mathcal{F}_{1}
$$

in the stationary case $\pi=\pi_{\mathrm{eq}}$,

$$
\widetilde{\xi}_{a, m}[f] \stackrel{\text { fidi }}{\longrightarrow} G_{\beta}^{\mathrm{eq}}[f], \quad f \in \mathcal{F}_{1} .
$$

Remark 1.5. The above theorem is related to a limit theorem for renewal counting processes which is the main result in [7]. Indeed, considering the parameterized class of functions $f_{t}=1_{[0, t]}, t \geq 0$, we have $\xi s_{a}^{-1}\left[f_{t}\right]=$ $\xi([0, a t])$. Applied to the equilibrium renewal model for which $\left.\mathbb{E}_{\mathrm{eq}} \xi^{i}([0, t])\right)=t / \mu$, this yields

$$
\widetilde{\xi}_{a, m}[f]=\frac{1}{b(a, m)} \sum_{i=1}^{m}\left(\xi^{i}([0, a t])-a t / \mu\right) .
$$


While $1_{[0, t]} \notin \mathcal{F}_{1}$ the Poisson integrals $J_{\beta}^{\mathrm{eq}}\left[1_{[0, t]}\right]$ and $J_{\beta}^{0}\left[1_{[0, t]}\right]$ are still well-defined. Put $J_{\beta}^{\mathrm{eq}}(t)=J_{\beta}^{\mathrm{eq}}\left[1_{[0, t]}\right]$ and $G_{\beta}^{\mathrm{eq}}(t)=G_{\beta}^{\mathrm{eq}}\left[1_{[0, t]}\right], t \geq 0$, that is

$$
J_{\beta}^{\mathrm{eq}}(t)=\int_{\mathbb{R} \times[0,+\infty)} \int_{0}^{t} 1_{[x, x+u]}(y) \mathrm{d} y \widetilde{N}(\mathrm{~d} x, \mathrm{~d} u),
$$

and

$$
G_{\beta}^{\mathrm{eq}}(t)=\int_{\mathbb{R} \times[0,+\infty)} \int_{0}^{t} 1_{[x, x+u]}(y) \mathrm{d} y \widetilde{W}(\mathrm{~d} x, \mathrm{~d} u), \quad t \geq 0 .
$$

According to [7], under assumption (ICR),

$$
\frac{1}{a} \sum_{i=1}^{m}\left(\xi^{i}([0, a t])-a t / \mu\right) \stackrel{f i d i}{\longrightarrow}-\frac{1}{\mu} c J_{\beta}^{\mathrm{eq}}(t / c),
$$

whereas under assumption (FCR),

$$
\frac{1}{\left(m \ell(a) a^{2-\beta}\right)^{1 / 2}} \sum_{i=1}^{m}\left(\xi^{i}([0, a t])-a t / \mu\right) \stackrel{f i d i}{\longrightarrow} G_{\beta}^{\mathrm{eq}}(t), \quad t \geq 0 .
$$

By construction, $G_{\beta}^{\mathrm{eq}}(t), t \geq 0$, is a Gaussian mean zero process with stationary increments and variance given by

$$
\int_{\mathbb{R} \times[0,+\infty)}\left(\int_{0}^{t} 1_{[x, x+u]}(y) \mathrm{d} y\right)^{2} n(\mathrm{~d} x, \mathrm{~d} u)=\frac{1}{\beta(1-\beta)} t^{2-\beta} .
$$

The normalized processes $B_{H}(t)=\sqrt{\beta(1-\beta)} G_{\beta}^{\mathrm{eq}}(t)$ and $P_{H}(t)=\sqrt{\beta(1-\beta)} J_{\beta}^{\mathrm{eq}}(t)$ have stationary increments and covariance function

$$
\operatorname{Cov}\left(B_{H}(t), B_{H}(s)\right)=\operatorname{Cov}\left(P_{H}(t), P_{H}(s)\right)=\frac{1}{2}\left(t^{2 H}+s^{2 H}-|t-s|^{2 H}\right) .
$$

The relation

$$
\mathbb{E}\left|B_{H}(t)-B_{H}(s)\right|^{2}=\mathbb{E}\left|P_{H}(t)-P_{H}(s)\right|^{2}=|t-s|^{2-\beta}
$$

with $\beta<1$ together with the Kolmogorov-Centsov criterion implies that there exists a modification of these processes with continuous sample paths. Thus, $B_{H}$ is a fractional Brownian motion with Hurst index $H=$ $1-\beta / 2 \in(1 / 2,1)$. By analogy, the mean zero non-Gaussian process $P_{H}$ has been called fractional Poisson motion, cf. $[2,5]$. By a refined analysis of higher-order moments it can be shown moreover that $P_{H}$ and $B_{H}$ share the same Hölder-regularity: in both cases the paths are $\gamma$-Hölder continuous of any order $\gamma<H$, [6].

\section{Asymptotics of moment measures}

This section is devoted to the proof of Theorem 1.1. We begin with some preliminaries.

\subsection{Preliminaries}

We give a simple criterion for the convergence $\stackrel{\mathcal{F}_{k}}{\longrightarrow}$ of signed Radon measures defined in (1.7). For $x=$ $\left(x_{1}, \ldots, x_{k}\right) \in[0,+\infty)^{k}$, we denote $[0, x]=\left[0, x_{1}\right] \times \ldots \times\left[0, x_{k}\right]$.

Lemma 2.1. Let $\left(m_{n}\right)_{n \geq 1}$ and $m$ be signed Radon measures. Suppose that the following conditions holds: for all $x \in[0,+\infty)^{k}$,

(i) $m_{n}([0, x]) \rightarrow m([0, x])$ as $n \rightarrow \infty$;

(ii) $\sup _{u \in[0, x]} \sup _{n \geq n_{0}}\left|m_{n}([0, u])\right|<\infty$ for some $n_{0} \geq 1$.

Then, $m_{n} \stackrel{\mathcal{F}_{k}}{\longrightarrow} m$ as $n \rightarrow \infty$. 
Proof. The proof is based on the following standard integration by part formula: for any signed Radon measure $m$ and $f \in \mathcal{F}_{k}$ with support included in $[0, x]$,

$$
m[f]=\int_{[0,+\infty)^{k}} f d m=(-1)^{k} \int_{[0, x]} \frac{\partial^{k} f}{\partial x_{1} \ldots \partial x_{k}}(u) m([0, u]) \mathrm{d} u .
$$

Using this, we have, as $n \rightarrow \infty$,

$$
\begin{aligned}
m_{n}[f] & =(-1)^{k} \int_{[0, x]} \frac{\partial^{k} f}{\partial x_{1} \ldots \partial x_{k}}(u) m_{n}([0, u]) \mathrm{d} u \\
& \longrightarrow(-1)^{k} \int_{[0, x]} \frac{\partial^{k} f}{\partial x_{1} \ldots \partial x_{k}}(u) m([0, u]) \mathrm{d} u=m[f] .
\end{aligned}
$$

The convergence is ensured by Lebesgue's dominated convergence theorem: condition (i) entails the pointwise convergence and (ii) the domination condition.

The following simple properties will be useful. A mapping $h:[0,+\infty)^{r} \rightarrow[0,+\infty)^{l}$ is said to be proper if any compact $K \subset[0,+\infty)^{l}$ has a compact preimage $h^{-1}(K) \subset[0,+\infty)^{r}$.

Lemma 2.2. Suppose that $\left(m_{n}\right)_{n \geq 1}$ and $m$ are signed Radon measure on $[0,+\infty)^{r}$ such that $m_{n} \stackrel{\mathcal{F}_{n}}{\longrightarrow} m$ as $n \rightarrow \infty$. Then

(1) for all smooth and proper functions $h:[0,+\infty)^{r} \rightarrow[0,+\infty)^{l}, m_{n} h^{-1} \stackrel{\mathcal{F}_{l}}{\longrightarrow} m h^{-1}$ as $n \rightarrow \infty$;

(2) for all $l \geq 1, m_{n} \otimes \lambda_{l} \stackrel{\mathcal{F}_{r+l}}{\longrightarrow} m \otimes \lambda_{l}$ as $n \rightarrow \infty$;

Proof. For the first point, it is enough to remark that if $f \in \mathcal{F}_{l}$ and $h$ is smooth and proper, then $f h \in \mathcal{F}_{r}$ so that

$$
\left(m_{n} h^{-1}\right)[f]=m_{n}[f h] \longrightarrow m[f h]=\left(m h^{-1}\right)[f] \quad \text { as } n \rightarrow \infty .
$$

For the second point, let $f \in \mathcal{F}_{r+l}$ and define the function $\tilde{f}$ by

$$
\tilde{f}\left(x_{1}, \ldots, x_{r}\right)=\int_{[0,+\infty)^{l}} f\left(x_{1}, \ldots, x_{r}, x_{r+1}, \ldots, x_{r+l}\right) \mathrm{d} x_{r+1} \ldots \mathrm{d} x_{r+l} .
$$

It is easy to show that $\tilde{f} \in \mathcal{F}_{r}$ so that

$$
\left(m_{n} \otimes \lambda_{l}\right)[f]=m_{n}[\tilde{f}] \longrightarrow m[\tilde{f}]=\left(m \otimes \lambda_{l}\right)[f] \quad \text { as } n \rightarrow \infty .
$$

\subsection{Asymptotics of factorial moment measures}

Before considering moment and centered moment measures, we need to consider factorial moment measures that have a simpler structure. The $k$ th factorial moment measure $M_{[k]}^{\pi}, k \geq 1$, is defined by

$$
M_{[k]}^{\pi}(A)=\mathbb{E}_{\pi}\left[\sum \delta_{\left(S_{n_{1}}, \ldots, S_{n_{k}}\right)}(A)\right], \quad A \in \mathcal{B}_{k},
$$

where the sum runs over the set of $k$-tuple's $\left(n_{1}, \ldots, n_{k}\right)$ of pairwise distinct non-negative integers, see [4].

To clarify the structure of factorial moment measures for the renewal point process $\xi$, we introduce the following additional notation. Let $\theta_{k}:[0,+\infty)^{k} \rightarrow[0,+\infty)^{k}$ be the injective mapping

$$
\theta_{k}\left(x_{1}, \ldots, x_{k}\right)=\left(x_{1}, x_{1}+x_{2}, \ldots, x_{1}+\ldots+x_{k}\right),
$$

and let $\Sigma_{k}$ denote the set of permutations of $\{1, \ldots, k\}$. A permutation $\sigma$ operates on $[0,+\infty)^{k}$ by permutation of the coordinates:

$$
\sigma\left(x_{1}, \ldots, x_{k}\right)=\left(x_{\sigma(1)}, \ldots, x_{\sigma(k)}\right), \quad\left(x_{1}, \ldots, x_{k}\right) \in[0,+\infty)^{k} .
$$


Lemma 2.3. For any $k \geq 1$, the $k$ th factorial moment measure $M_{[k]}^{\pi}$ satisfies

$$
M_{[k]}^{\pi}=\sum_{\sigma \in \Sigma_{k}}\left[(\pi+\pi * U) \otimes U^{\otimes(k-1)}\right] \theta_{k}^{-1} \sigma^{-1},
$$

with $U=\sum_{n=1}^{\infty} F^{* n}$.

Proof. By reindexing the terms in the sum (2.1),

$$
\begin{aligned}
M_{[k]}^{\pi}(A) & =\mathbb{E}_{\pi} \sum_{\sigma \in \Sigma_{k}} \sum_{0 \leq n_{1}<\ldots<n_{k}} \delta_{\left(S_{n_{\sigma(1)}}, \ldots, S_{n_{\sigma(k)}}\right)}(A) \\
& =\mathbb{E}_{\pi} \sum_{\sigma \in \Sigma_{k}} \sum_{0 \leq n_{1}<\ldots<n_{k}} \delta_{\left(S_{n_{1}}, \ldots, S_{n_{k}}\right)}\left(\sigma^{-1}(A)\right) \\
& =\sum_{\sigma \in \Sigma_{k}} \sum_{0 \leq n_{1}<\ldots<n_{k}} \mathbb{P}_{\pi}\left[\left(S_{n_{1}}, \ldots, S_{n_{k}}\right) \in \sigma^{-1}(A)\right] .
\end{aligned}
$$

Then, for all $\sigma \in \Sigma_{k}$, by changing the summation indices according to $i_{1}=n_{1} \geq 0, i_{2}=n_{2}-n_{1} \geq 1, \ldots, i_{k}=$ $n_{k}-n_{k-1} \geq 1$,

$\sum_{0 \leq n_{1}<\ldots<n_{k}} \mathbb{P}_{\pi}\left[\left(S_{n_{1}}, \ldots, S_{n_{k}}\right) \in \sigma^{-1}(A)\right]=\sum_{i_{1} \geq 0} \sum_{i_{2}>0, \ldots, i_{k}>0} \mathbb{P}_{\pi}\left[\left(S_{n_{1}}, S_{n_{2}}-S_{n_{1}}, \ldots, S_{n_{k}}-S_{n_{k-1}}\right) \in \theta_{k}^{-1} \sigma^{-1}(A)\right]$.

Since the law of $\left(S_{n_{1}}, S_{n_{2}}-S_{n_{1}}, \ldots, S_{n_{k}}-S_{n_{k-1}}\right)$ is equal to $\left(\pi * F^{* i_{1}}\right) \otimes F^{* i_{2}} \otimes \ldots \otimes F^{* i_{k}}$ with $F^{* 0}=\delta_{0}$, we get

$$
\begin{aligned}
\sum_{0 \leq n_{1}<\ldots<n_{k}} \mathbb{P}_{\pi}\left[\left(S_{n_{1}}, \ldots, S_{n_{k}}\right) \in \sigma^{-1}(A)\right] & =\sum_{i_{1} \geq 0} \sum_{i_{2}>0, \ldots, i_{k}>0}\left[\left(\pi * F^{* i_{1}}\right) \otimes F^{* i_{2}} \otimes \ldots \otimes F^{* i_{k}}\right]\left(\theta_{k}^{-1} \sigma^{-1} A\right) \\
& =\left[(\pi+\pi * U) \otimes U^{\otimes(k-1)}\right]\left(\theta_{k}^{-1} \sigma^{-1} A\right)
\end{aligned}
$$

and this proves the desired result.

We are now in position to consider scaled factorial moment measures. At this stage we recall the basic assumption (1.2) that the inter-renewal distribution has a regularly varying decay with tail parameter $1+\beta$ as well as the notion of $\mathcal{F}_{k}$-convergence introduced in (1.7).

\section{Lemma 2.4.}

In the pure case when $\pi=\delta_{0}$, we have as $a \rightarrow \infty$,

$$
\frac{M_{[k]}^{0} s_{a}^{-1}-(a / \mu)^{k} \lambda_{k}}{a^{k-\beta} \ell(a)} \stackrel{\mathcal{F}_{k}}{\longrightarrow} \frac{1}{\beta \mu^{k+1}} P_{k}^{0}, \quad k \geq 1 .
$$

In the stationary case when $\pi=\pi_{\mathrm{eq}}$, as $a \rightarrow \infty$,

$$
\frac{M_{[k]}^{\mathrm{eq}} s_{a}^{-1}-(a / \mu)^{k} \lambda_{k}}{a^{k-\beta} \ell(a)} \stackrel{\mathcal{F}_{k}}{\longrightarrow} \frac{1}{\beta \mu^{k+1}} P_{k}^{\mathrm{eq}}, \quad k \geq 1 .
$$

Proof. Since the measure $\lambda_{k} \theta_{k}^{-1}$ is absolutely continuous with respect to $\lambda_{k}$ with density $1_{\left\{y_{1} \leq \ldots \leq y_{k}\right\}}$, we have

$$
\sum_{\sigma \in \Sigma_{k}} \lambda_{k} \theta_{k}^{-1} \sigma^{-1}=\lambda_{k}
$$


By Lemma 2.3,

$$
\begin{aligned}
\frac{M_{[k]}^{\pi} s_{a}^{-1}-(a / \mu)^{k} \lambda_{k}}{a^{k-\beta} \ell(a)} & =\frac{1}{a^{k-\beta} \ell(a)}\left(M_{[k]}^{\pi}-\mu^{-k} \lambda_{k}\right) s_{a}^{-1} \\
& =\frac{1}{a^{k-\beta} \ell(a)} \sum_{\sigma \in \Sigma_{k}}\left((\pi+\pi * U) \otimes U^{\otimes k-1}-\mu^{-k} \lambda_{k}\right) \theta_{k}^{-1} \sigma^{-1} s_{a}^{-1} .
\end{aligned}
$$

Using the commutation relations $s_{a} \sigma=\sigma s_{a}$ and $s_{a} \theta_{k}=\theta_{k} s_{a}$, this implies

$$
\frac{M_{[k]}^{\pi} s_{a}^{-1}-(a / \mu)^{k} \lambda_{k}}{a^{k-\beta} \ell(a)}=\sum_{\sigma \in \Sigma_{k}}\left(\frac{\left((\pi+\pi * U) \otimes U^{\otimes(k-1)}\right) s_{a}^{-1}-(a / \mu)^{k} \lambda_{k}}{a^{k-\beta} \ell(a)}\right) \theta_{k}^{-1} \sigma^{-1} .
$$

The further analysis of (2.2) will be carried out separately for the pure and the stationary cases.

We first consider the pure case. For $k=1$, we use the classical result for the renewal function $U([0, x])=$ $\mathbb{E}_{0} \xi([0, x])$ with heavy-tailed inter-renewal distribution that satisfies assumption (1.2) (see Thm. 4 in $[20]$ ):

$$
U([0, x])-\frac{x}{\mu} \sim \frac{\ell(x) x^{1-\beta}}{\mu^{2} \beta(1-\beta)} \quad \text { as } x \rightarrow \infty .
$$

For $k=1$ we have $M_{[1]}^{0}=\delta_{0}+U$ and, for $x>0, P_{1}^{0}([0, x])=\frac{1}{1-\beta} x^{1-\beta}$. This entails condition (i) of Lemma 2.1: for $x>0$ and as $a \rightarrow \infty$,

$$
\left(\frac{M_{[1]}^{0} s_{a}^{-1}-a / \mu \lambda_{1}}{a^{1-\beta} \ell(a)}\right)([0, x])=\frac{U([0, a x])+1-a x / \mu}{a^{1-\beta} \ell(a)} \longrightarrow \frac{x^{1-\beta}}{\mu^{2} \beta(1-\beta)}=\frac{1}{\beta \mu^{2}} P_{1}^{0}([0, x]) .
$$

Condition (ii) of Lemma 2.1 is the property that there exists $a_{0}>0$ such that

$$
\sup _{u \in[0, x]} \sup _{a \geq a_{0}} \frac{U([0, a u])-a u / \mu}{a^{1-\beta} \ell(a)}<\infty .
$$

The proof of (2.4) relies on the theory of regularly varying functions and is more technical; it is postponed to Appendix A.

Turning to the case $k \geq 2$ and considering (2.2) with $\pi=\delta_{0}$, we will prove the $\mathcal{F}_{k}$-convergence of measures

$$
\frac{\left(\left(\delta_{0}+U\right) \otimes U^{\otimes(k-1)}\right) s_{a}^{-1}-(a / \mu)^{k} \lambda_{k}}{a^{k-\beta} \ell(a)} \stackrel{\mathcal{F}_{k}}{\longrightarrow} \frac{1}{\beta \mu^{k+1}} Q_{k}^{0}, \quad \text { as } a \rightarrow \infty,
$$

where $\left(d Q_{k}^{0} / \mathrm{d} \lambda_{k}\right)(x)=\sum_{i=1}^{k} x_{i}^{-\beta}$. To this end, we note that for $x=\left(x_{1}, \ldots, x_{k}\right) \in[0,+\infty)^{k}$,

$$
Q_{k}^{0}([0, x])=\frac{1}{1-\beta}\left(\prod_{i=1}^{k} x_{j}\right)\left(\sum_{i=1}^{k} x_{i}^{-\beta}\right)
$$

and, using (2.3),

$$
\begin{aligned}
\left(\left(\delta_{0}+U\right) \otimes U^{\otimes(k-1)}\right)([0, a x]) & =\left(1+U\left(\left[0, a x_{1}\right]\right) \prod_{i=2}^{k} U\left(\left[0, a x_{i}\right]\right)\right. \\
& =\prod_{i=1}^{k}\left(a x_{i} / \mu+\frac{\ell\left(a x_{i}\right)\left(a x_{i}\right)^{1-\beta}}{\mu^{2} \beta(1-\beta)}+o\left(\ell(a) a^{1-\beta}\right)\right) \\
& =(a / \mu)^{k}\left(\prod_{i=1}^{k} x_{i}\right)\left(1+\frac{\ell(a) a^{-\beta}}{\mu \beta(1-\beta)} \sum_{i=1}^{k} x_{i}^{-\beta}+o\left(\ell(a) a^{-\beta}\right)\right) \\
& =\frac{a^{k}}{\mu^{k}} \lambda_{k}([0, x])+\frac{a^{k-\beta} \ell(a)}{\beta \mu^{k+1}} Q_{k}^{0}([0, x])+o\left(\ell(a) a^{k-\beta}\right),
\end{aligned}
$$


which proves condition (i) of Lemma 2.1. Equations (2.4) and (2.6) together imply condition (ii), i.e.,

$$
\sup _{u \in[0, x]} \sup _{a \geq a_{0}} \frac{\left(\left(\delta_{0}+U\right) \otimes U^{\otimes(k-1)}\right)([0, a x])-(a / \mu)^{k} \lambda_{k}([0, x])}{a^{k-\beta} \ell(a)}<\infty
$$

and hence the $\mathcal{F}_{k}$-convergence (2.5) follows from Lemma 2.1. The mapping $\sigma \theta_{k}$ is smooth and proper and so (2.2) and (2.5) together with Lemma 2.2 entail

$$
\frac{M_{[k]}^{0} s_{a}^{-1}-(a / \mu)^{k} \lambda_{k}}{a^{k-\beta} \ell(a)} \stackrel{\mathcal{F}_{k}}{\longrightarrow} \frac{1}{\beta \mu^{k+1}} \sum_{\sigma \in \Sigma_{k}} Q_{k}^{0} \theta_{k}^{-1} \sigma^{-1}
$$

It remains to verify that the measure $\sum_{\sigma \in \Sigma_{k}} Q_{k}^{0} \theta_{k}^{-1} \sigma^{-1}$ equals $P_{k}^{0}$. For this we observe that $Q_{k}^{0} \theta_{k}^{-1}$ is absolutely continuous with respect to $\lambda_{k}$, with density

$$
\frac{\mathrm{d}\left(Q_{k}^{0} \theta_{k}^{-1}\right)}{\mathrm{d} \lambda_{k}}(y)=\left(\left|y_{1}\right|^{-\beta}+\sum_{i=2}^{k}\left|y_{i}-y_{i-1}\right|^{-\beta}\right) 1_{\left\{y_{1} \leq \ldots \leq y_{k}\right\}} .
$$

Hence the measure $\sum_{\sigma \in \Sigma_{k}} Q_{k}^{0} \theta_{k}^{-1} \sigma^{-1}$ has the density function

$$
\sum_{\sigma \in \Sigma_{k}}\left(\left|y_{\sigma(1)}\right|^{-\beta}+\sum_{i=2}^{k}\left|y_{\sigma(i)}-y_{\sigma(i-1)}\right|^{-\beta}\right) 1_{\left\{y_{\sigma(1)} \leq \ldots \leq y_{\sigma(k)}\right\}}=\left|y_{(1)}\right|^{-\beta}+\sum_{i=2}^{k}\left|y_{(i)}-y_{(i-1)}\right|^{-\beta}=\frac{\mathrm{d} P_{k}^{0}}{\mathrm{~d} \lambda_{k}}(y) .
$$

Taking into account the property (2.4) of regularly varying functions in Appendix A, this completes the proof of the lemma for the pure case.

The proof in the stationary case is quite similar and is only given in brief. For $k=1$,

$$
M_{[1]}^{\mathrm{eq}}=\pi_{\mathrm{eq}}+\pi_{\mathrm{eq}} * U=\lambda_{1} / \mu
$$

hence

$$
M_{[1]}^{\mathrm{eq}}-\lambda_{1} / \mu=P_{1}^{\mathrm{eq}}=0
$$

For $k \geq 2$, equation (2.2) yields

$$
\frac{M_{[k]}^{\mathrm{eq}} s_{a}^{-1}-(a / \mu)^{k} \lambda_{k}}{a^{k-\beta} \ell(a)}=\sum_{\sigma \in \Sigma_{k}}\left(\frac{\left(\mu^{-1} \lambda_{1} \otimes U^{\otimes(k-1)}\right) s_{a}^{-1}-(a / \mu)^{k} \lambda_{k}}{a^{k-\beta} \ell(a)}\right) \theta_{k}^{-1} \sigma^{-1} .
$$

Similarly as in (2.5), we then prove

$$
\frac{\left(\mu^{-1} \lambda_{1} \otimes U^{\otimes(k-1)}\right) s_{a}^{-1}-(a / \mu)^{k} \lambda_{k}}{a^{k-\beta} \ell(a)} \stackrel{\mathcal{F}_{k}}{\longrightarrow} \frac{1}{\beta \mu^{k+1}} Q_{k}^{\mathrm{eq}}
$$

with $\left(\mathrm{d} Q_{k}^{\mathrm{eq}} / \mathrm{d} \lambda_{k}\right)(x)=\sum_{i=2}^{k} x_{i}^{-\beta}$. The desired result now follows from the fact that

$$
\sum_{\sigma \in \Sigma_{k}} Q_{k}^{\mathrm{eq}} \theta_{k}^{-1} \sigma^{-1}=P_{k}^{\mathrm{eq}}
$$




\subsection{Asymptotics of moment measures}

Moment measures can be expressed in terms of factorial moment measures. Trivially, for $k=1, M_{1}^{\pi}=M_{[1]}^{\pi}$. For $k=2$ and $A_{1}, A_{2} \in \mathcal{B}_{1}$ :

$$
\begin{aligned}
M_{2}^{\pi}\left(A_{1} \times A_{2}\right) & =\mathbb{E}_{\pi}\left[\sum_{n_{1}, n_{2} \geq 0} \delta_{\left(S_{n_{1}}, S_{n_{2}}\right)}\left(A_{1} \times A_{2}\right)\right] \\
& =\mathbb{E}_{\pi}\left[\sum_{n_{1} \neq n_{2}} \delta_{\left(S_{n_{1}}, S_{n_{2}}\right)}\left(A_{1} \times A_{2}\right)\right]+\mathbb{E}_{\pi}\left[\sum_{n \geq 0} \delta_{\left(S_{n}, S_{n}\right)}\left(A_{1} \times A_{2}\right)\right] \\
& =M_{[2]}^{\pi}\left(A_{1} \times A_{2}\right)+M_{[1]}^{\pi}\left(A_{1} \cap A_{2}\right) .
\end{aligned}
$$

Define $i_{12}:[0,+\infty) \rightarrow[0,+\infty)^{2}$ given by $i_{12}(x)=(x, x)$. We have $i_{12}^{-1}\left(A_{1} \times A_{2}\right)=A_{1} \cap A_{2}$ so that

$$
M_{2}^{\pi}=M_{[2]}^{\pi}+M_{[1]}^{\pi} i_{1,2}^{-1} .
$$

When $k=3$, a similar computation yields

$$
\begin{aligned}
M_{3}^{\pi}\left(A_{1} \times A_{2} \times A_{3}\right)= & M_{[3]}^{\pi}\left(A_{1} \times A_{2} \times A_{3}\right)+M_{[2]}^{\pi}\left(\left(A_{1} \cap A_{2}\right) \times A_{3}\right)+M_{[2]}^{\pi}\left(\left(A_{1} \cap A_{3}\right) \times A_{2}\right) \\
& +M_{[2]}^{\pi}\left(\left(A_{2} \cap A_{3}\right) \times A_{1}\right)+M_{[1]}^{\pi}\left(A_{1} \cap A_{2} \cap A_{3}\right) .
\end{aligned}
$$

Define the mappings $i_{12,3}(x, y)=(x, x, y), i_{13,2}(x, y)=(x, y, x), i_{23,1}(x, y)=(y, x, x)$ and $i_{123}(x)=(x, x, x)$. Then,

$$
M_{3}^{\pi}=M_{[3]}^{\pi}+M_{[2]}^{\pi} i_{12,3}^{-1}+M_{[2]}^{\pi} i_{13,2}^{-1}+M_{[2]}^{\pi} i_{23,1}^{-1}+M_{[1]}^{\pi} i_{123}^{-1} .
$$

In the general case $k \geq 1$, the combinatorial relation between moment measures and factorial moment measures can be found in [4], Exercise 5.4.5, page 143. For $1 \leq j \leq k$, let $\mathcal{P}_{j k}$ be the set of all partitions of $\{1, \ldots, k\}$ into $j$ nonempty subsets. An element $\mathcal{T}=\left\{S_{1}(\mathcal{T}), \ldots, S_{j}(\mathcal{T})\right\} \in \mathcal{P}_{j k}$ formed by $j$ disjoint subsets labelled in arbitrary order is called a $j$-partition. The cardinality of the subset $S_{i}(\mathcal{T})$ is denoted by $\left|S_{i}(\mathcal{T})\right|$ so that $\sum_{i=1}^{j}\left|S_{i}(\mathcal{T})\right|=k$. For any $j$-partition $\mathcal{T} \in \mathcal{P}_{j k}$, the injection $i_{\mathcal{T}}:[0,+\infty)^{j} \rightarrow[0,+\infty)^{k}$ is defined by $i_{\mathcal{T}}\left(x_{1}, \ldots, x_{j}\right)=\left(y_{1}, \ldots, y_{k}\right)$ where $y_{p}=x_{i}$ if $p \in S_{i}(\mathcal{T})$. Then

$$
M_{k}^{\pi}=\sum_{j=1}^{k} \sum_{\mathcal{T} \in \mathcal{P}_{j k}} M_{[j]}^{\pi} i_{\mathcal{T}}^{-1} .
$$

\section{Proof of Theorem 1.1. Moment measures.}

To prove Theorem 1.1 as regards properties of the moment measures $M_{k}^{0}$ and $M_{k}^{\mathrm{eq}}$, we will show that factorial moment measures and moment measures share the same asymptotic behavior. More precisely, using (2.7),

$$
\frac{M_{k}^{\pi} s_{a}^{-1}-(a / \mu)^{k} \lambda_{k}}{a^{k-\beta} \ell(a)}=\frac{M_{[k]}^{\pi} s_{a}^{-1}-(a / \mu)^{k} \lambda_{k}}{a^{k-\beta} \ell(a)}+\sum_{j=1}^{k-1} \sum_{\mathcal{T} \in \mathcal{P}_{j k}} \frac{M_{[j]}^{\pi} i_{\mathcal{T}}^{-1} s_{a}^{-1}}{a^{k-\beta} \ell(a)} .
$$

We have shown in Lemma 2.4 that the factorial moments in the first term on the right hand side $\mathcal{F}_{k}$-converge to the desired limit measures. To complete the proof it suffices to verify that the second summation term vanishes in the limit:

$$
\sum_{j=1}^{k-1} \sum_{\mathcal{T} \in \mathcal{P}_{j k}} \frac{M_{[j]}^{\pi} i_{\mathcal{T}}^{-1} s_{a}^{-1}}{a^{k-\beta} \ell(a)} \stackrel{\mathcal{F}_{k}}{\longrightarrow} 0 .
$$

We will establish (2.8) for the pure case $\pi=\delta_{0}$. A straightforward adaptation yields the stationary case. By Lemma 2.4, for $1 \leq j \leq k-1$,

$$
\frac{M_{[j]}^{0} s_{a}^{-1}}{(a / \mu)^{j}} \stackrel{\mathcal{F}_{j}}{\longrightarrow} \lambda_{j}
$$


and hence

$$
\frac{M_{[j]}^{0} s_{a}^{-1}}{a^{k-\beta} \ell(a)} \stackrel{\mathcal{F}_{j}}{\longrightarrow} 0 .
$$

For $\mathcal{T} \in \mathcal{P}_{j k}$, the mapping $i_{\mathcal{T}}$ is smooth and proper and such that $s_{a} i_{\mathcal{T}}=i_{\mathcal{T}} s_{a}$. Hence, Lemma 2.2 (1) implies

$$
\frac{M_{[j]}^{0} i_{\mathcal{T}}^{-1} s_{a}^{-1}}{a^{k-\beta} \ell(a)} \stackrel{\mathcal{F}_{k}}{\longrightarrow} 0
$$

\subsection{Asymptotics of centered moment measures}

To prove the results in Theorem 1.1 related to centered moment measures we will apply a formula for centered $k$-moment measures in terms of $m$-moment measures of order $m \leq k$. First of all, we have $\widetilde{M}_{1}^{\pi}=M_{1}^{\pi}-\lambda_{1} / \mu$.

Lemma 2.5. For $k \geq 2$, consider cylinder sets $A=\otimes_{i=1}^{k} A_{i}$, where $A_{1}, \ldots, A_{k} \in \mathcal{B}_{1}$. For any subset $I$ of indices, $I \subset\{1, \ldots, k\}$, denote $A_{I}=\underset{i \in I}{\otimes} A_{i}$ and $A_{I^{c}}=\underset{i \notin I}{\otimes} A_{i}$. Then

$$
\widetilde{M}_{k}^{\pi}(A)=\sum_{r=1}^{k} \sum_{|I|=r}(-1)^{k-r}\left(M_{r}^{\pi}-\frac{\lambda_{r}}{\mu^{r}}\right)\left(A_{I}\right) \frac{\lambda_{k-r}\left(A_{I^{c}}\right)}{\mu^{k-r}}
$$

Proof. We compute

$$
\begin{aligned}
\widetilde{M}_{k}^{\pi}(A) & =\mathbb{E}_{\pi}\left[\prod_{i=1}^{k}\left(\xi\left(A_{i}\right)-\lambda_{1}\left(A_{i}\right) / \mu\right)\right] \\
& =\mathbb{E}_{\pi}\left[\sum_{I \subset\{1, \ldots, k\}}(-1)^{k-|I|}\left(\prod_{i \in I} \xi\left(A_{i}\right)\right)\left(\prod_{i \notin I} \lambda_{1}\left(A_{i}\right) / \mu\right)\right] \\
& =\sum_{I \subset\{1, \ldots, k\}}(-1)^{k-|I|} M_{|I|}^{\pi}\left(\otimes_{i \in I} A_{i}\right) \frac{\lambda_{k-|I|}\left(\otimes_{i \notin I} A_{i}\right)}{\mu^{k-|I|}}
\end{aligned}
$$

which may be written

$$
\widetilde{M}_{k}^{\pi}(A)=\sum_{r=0}^{k} \sum_{|I|=r}(-1)^{k-r} M_{r}^{\pi}\left(A_{I}\right) \frac{\lambda_{k-r}\left(A_{I^{c}}\right)}{\mu^{k-r}}
$$

Observe that

$$
\sum_{r=0}^{k} \sum_{|I|=r}(-1)^{k-r}=\sum_{r=0}^{k}(-1)^{k-r}\left(\begin{array}{l}
k \\
r
\end{array}\right)=(1-1)^{k}=0 .
$$

This implies

$$
\sum_{r=0}^{k} \sum_{|I|=r}(-1)^{k-r} \frac{\lambda_{r}\left(A_{I}\right)}{\mu^{r}} \frac{\lambda_{k-r}\left(A_{I^{c}}\right)}{\mu^{k-r}}=\frac{\lambda_{k}(A)}{\mu^{k}} \sum_{r=0}^{k} \sum_{|I|=r}(-1)^{k-r}=0 .
$$

Taking the difference between (2.9) and (2.10), the term with $r=0$ cancels out and the result follows.

Proof of Theorem 1.1. Centered moment measures.

To prove the remaining statements of Theorem 1.1 devoted to centered moment measures $\widetilde{M}_{k}^{0}$ and $\widetilde{M}_{k}^{\text {eq }}$, we apply Lemma 2.5 to obtain

$$
\frac{\widetilde{M}_{k}^{\pi} s_{a}^{-1}(A)}{a^{k-\beta} \ell(a)}=(-1)^{k} \sum_{r \geq 1} \sum_{|I|=r}(-1)^{r} \frac{\left(M_{r}^{\pi} s_{a}^{-1}-(a / \mu)^{r} \lambda_{r}\right)\left(A_{I}\right)}{a^{r-\beta} \ell(a)} \frac{\lambda_{k-r}\left(A_{I^{c}}\right)}{\mu^{k-r}} .
$$


Hence, with the notation $x=\left(x_{1}, \ldots, x_{k}\right), x_{I}=\left(x_{i}\right)_{i \in I}$ and $x_{I^{c}}=\left(x_{i}\right)_{i \in I}$,

$$
\left(\frac{\widetilde{M}_{k}^{\pi} s_{a}^{-1}}{a^{k-\beta} \ell(a)}\right)(\mathrm{d} x)=(-1)^{k} \sum_{r \geq 1} \sum_{|I|=r}(-1)^{r}\left(\frac{\left(M_{r}^{\pi} s_{a}^{-1}-(a / \mu)^{r} \lambda_{r}\right)}{a^{r-\beta} \ell(a)}\right)\left(\mathrm{d} x_{I}\right)\left(\frac{\lambda_{k-r}\left(A_{I^{c}}\right)}{\mu^{k-r}}\right)\left(\mathrm{d} x_{I^{c}}\right) .
$$

This brings us in position to apply the already established part of Theorem 1.1. For $\pi=\delta_{0}$ we have shown, for all $r \geq 1$,

$$
\frac{M_{r}^{0}-(a / \mu)^{r} \lambda_{r}}{a^{r-\beta} \ell(a)} \stackrel{\mathcal{F}_{r}}{\longrightarrow} \frac{1}{\beta \mu^{r+1}} P_{r}^{0}, \quad \text { as } a \rightarrow \infty .
$$

In view of Lemma $2.2(2)$ with $\ell=k-r$,

$$
\left(\frac{M_{r}^{0}-(a / \mu)^{r} \lambda_{r}}{a^{r-\beta} \ell(a)}\right)\left(\mathrm{d} x_{I}\right) \lambda_{k-r}\left(\mathrm{~d} x_{I^{c}}\right) \stackrel{\mathcal{F}_{k}}{\longrightarrow}\left(\frac{1}{\beta \mu^{r+1}} P_{r}^{0}\right)\left(\mathrm{d} x_{I}\right) \lambda_{k-r}\left(\mathrm{~d} x_{I^{c}}\right), \quad \text { as } a \rightarrow \infty,
$$

and equation (2.11) entails

$$
\left(\frac{\widetilde{M}_{k}^{0} s_{a}^{-1}}{a^{k-\beta} \ell(a)}\right)(\mathrm{d} x) \stackrel{\mathcal{F}_{k}}{\longrightarrow} \frac{(-1)^{k}}{\beta \mu^{k+1}} \sum_{r=1}^{k} \sum_{|I|=r}(-1)^{r}\left(\frac{1}{\beta \mu^{r+1}} P_{r}^{0}\right)\left(\mathrm{d} x_{I}\right) \lambda_{k-r}\left(\mathrm{~d} x_{I^{c}}\right), \quad \text { as } a \rightarrow \infty .
$$

When $\pi=\pi_{\text {eq }}$, all terms in (2.11) with $r=1$ vanish. For this case, similar computations show

$$
\left(\frac{\widetilde{M}_{k}^{\mathrm{eq}} s_{a}^{-1}}{a^{k-\beta} \ell(a)}\right)(\mathrm{d} x) \stackrel{\mathcal{F}_{k}}{\longrightarrow} \frac{(-1)^{k}}{\beta \mu^{k+1}} \sum_{r=2}^{k} \sum_{|I|=r}(-1)^{r}\left(\frac{1}{\beta \mu^{r+1}} P_{r}^{\mathrm{eq}}\right)\left(\mathrm{d} x_{I}\right) \lambda_{k-r}\left(\mathrm{~d} x_{I^{c}}\right), \quad a \rightarrow \infty .
$$

It remains to identify the limiting centered measures as $\widetilde{P}_{k}^{0}$ and $\widetilde{P}_{k}^{\text {eq }}$ introduced in (1.4) and (1.5), i.e. we must prove

$$
\sum_{r=1}^{k} \sum_{|I|=r}(-1)^{r} P_{r}^{0}\left(\mathrm{~d} x_{I}\right) \lambda_{k-r}\left(\mathrm{~d} x_{I^{c}}\right)=\widetilde{P}_{k}^{0}(\mathrm{~d} x)
$$

and

$$
\sum_{r=2}^{k} \sum_{|I|=r}(-1)^{r} P_{r}^{\mathrm{eq}}\left(\mathrm{d} x_{I}\right) \lambda_{k-r}\left(\mathrm{~d} x_{I^{c}}\right)=\widetilde{P}_{k}^{\mathrm{eq}}(\mathrm{d} x)
$$

We begin with (2.13), which is the same as

$$
\sum_{r=2}^{k} \sum_{|I|=r}(-1)^{r} \frac{\mathrm{d} P_{r}^{\mathrm{eq}}}{\mathrm{d} \lambda_{r}}\left(x_{I}\right)=\frac{\mathrm{d} \widetilde{P}_{k}^{\mathrm{eq}}}{\mathrm{d} \lambda_{k}}(x)
$$

since $P_{r}^{\text {eq }}$ is absolutely continuous with respect to Lebesgue measure $\lambda_{r}$ with density (1.4). As both sides of the equality are symmetric functions of $x=\left(x_{1}, \ldots, x_{k}\right)$, it is enough to prove (2.14) for the case $x_{1} \leq \ldots \leq x_{k}$. Then, if $I=\left\{i_{1}, \ldots, i_{r}\right\}$ with $i_{1}<\ldots<i_{r}$,

$$
\frac{\mathrm{d} P_{r}^{\mathrm{eq}}}{\mathrm{d} \lambda_{r}}\left(x_{I}\right)=\sum_{j=2}^{r}\left|x_{i_{j}}-x_{i_{j-1}}\right|^{-\beta}
$$

and

$$
\sum_{r=2}^{k} \sum_{|I|=r}(-1)^{r} \frac{\mathrm{d} P_{r}^{\mathrm{eq}}}{\mathrm{d} \lambda_{r}}\left(x_{I}\right)=\sum_{r=2}^{k} \sum_{1 \leq i_{1}<\ldots<i_{r} \leq k}(-1)^{r} \sum_{j=2}^{r}\left|x_{i_{j}}-x_{i_{j-1}}\right|^{-\beta}=\sum_{1 \leq l_{1}<l_{2} \leq k} c_{l_{1}, l_{2}}\left|x_{l_{2}}-x_{l_{1}}\right|^{-\beta},
$$


for suitable coefficients $c_{l_{1}, l_{2}}$ obtained by reordering terms. We claim that $c_{1, k}=1$ and $c_{l_{1}, l_{2}}=0$ if $\left(l_{1}, l_{2}\right) \neq(1, k)$, so that

$$
\sum_{1 \leq l_{1}<l_{2} \leq k} c_{l_{1}, l_{2}}\left|x_{l_{2}}-x_{l_{1}}\right|^{-\beta}=\left|x_{k}-x_{1}\right|^{-\beta}
$$

which is the desired representation (2.14). To compute the coefficients $c_{l_{1}, l_{2}}$, we write $c_{l_{1}, l_{2}}=\sum(-1)^{r}$, with the sum running over all $r$-tuplets $i_{1}<\ldots<i_{r}, 2 \leq r \leq k$, such that $l_{2}=i_{j}$ and $l_{1}=i_{j-1}$ for some $j \in\{2, \ldots, r\}$. Such $r$-tuplets are exactly those containing $l_{1}$ and $l_{2}$ but no elements inbetween. They are hence obtained by choosing $r-2$ indices in $\{1, \ldots, k\} \backslash\left\{l_{1}, \ldots, l_{2}\right\}$, which can be done in exactly $\left(\begin{array}{c}k-l_{2}+l_{1}-1 \\ r-2\end{array}\right)$ ways. By convention, the binomial coefficient is 0 if $r-2>k-l_{2}+l_{1}-1$. Finally, using the binomial formula,

$$
c_{l_{1}, l_{2}}=\sum_{r=2}^{k}\left(\begin{array}{c}
k-l_{2}+l_{1}-1 \\
r-2
\end{array}\right)(-1)^{r}=(1-1)^{k-l_{2}+l_{1}-1},
$$

which verifies that we have indeed $c_{1, k}=1$ and $c_{l_{1}, l_{2}}=0$ if $\left(l_{1}, l_{2}\right) \neq(1, k)$.

We now consider (2.12) which is equivalent to

$$
\sum_{r=1}^{k} \sum_{|I|=r}(-1)^{r} \frac{\mathrm{d} P_{r}^{0}}{\mathrm{~d} \lambda_{r}}\left(x_{I}\right)=\frac{\mathrm{d} \widetilde{P}_{k}^{0}}{\mathrm{~d} \lambda_{k}}(x)
$$

It is enough to consider the case $x_{1} \leq \ldots \leq x_{k}$. If we take $I=\left\{i_{1}, \ldots, i_{r}\right\}$ with $i_{1}<\ldots<i_{r}$ and use for convenience $i_{0}=0$ and $x_{0}=0$, then

$$
\sum_{r=1}^{k} \sum_{|I|=r}(-1)^{r} \frac{\mathrm{d} P_{r}^{0}}{\mathrm{~d} \lambda_{r}}\left(x_{I}\right)=\sum_{r=1}^{k} \sum_{1 \leq i_{1}<\ldots<i_{r} \leq k}(-1)^{r} \sum_{j=1}^{r}\left|x_{i_{j}}-x_{i_{j-1}}\right|^{-\beta} .
$$

Reordering terms, this is

$$
\sum_{0 \leq l_{1}<l_{2} \leq k} c_{l_{1}, l_{2}}^{\prime}\left|x_{l_{2}}-x_{l_{1}}\right|^{-\beta}
$$

for suitable coefficients $c_{l_{1}, l_{2}}^{\prime}$. Again we will prove that most of the coefficients vanish except $c_{1, k}^{\prime}=1$ and $c_{0, k}^{\prime}=-1$ so that the corresponding density is $\left|x_{(k)}-x_{(1)}\right|^{-\beta}-\left|x_{(k)}\right|^{-\beta}$, which is (2.15). To see this we observe that for the case $1 \leq l_{1}<l_{2} \leq k$, the previous computations remain the same with $c_{l_{1}, l_{2}}^{\prime}=c_{l_{1}, l_{2}}$ equal to 1 if $\left(l_{1}, l_{2}\right)=(1, k)$ and 0 otherwise. The case $l_{1}=0$ needs to be considered separately. Here, $c_{0, l_{2}}^{\prime}=\sum_{I}(-1)^{r}$ with the sum running over all $r$-tuplets $i_{1}<\ldots<i_{r}, 1 \leq r \leq k$, such that $l_{2}=i_{1}$. There exists exactly $\left(\begin{array}{c}k-l_{2} \\ r-1\end{array}\right)$ such $r$-tuplets, obtained by choosing $r-1$ indices in the set $\left\{l_{2}+1, \ldots, k\right\}$. Finally,

$$
c_{0, l_{2}}^{\prime}=\sum_{r=1}^{k}\left(\begin{array}{c}
k-l_{2} \\
r-1
\end{array}\right)(-1)^{r}=-\sum_{r=0}^{k-l_{2}}\left(\begin{array}{c}
k-l_{2} \\
r
\end{array}\right)(-1)^{r}=-(1-1)^{k-l_{2}},
$$

which is 0 if $l_{2}<k$ and -1 if $l_{2}=k$.

\section{Aggregation of sources}

The Proof of Theorem 1.4 is based on the method of cumulants [8], a variant of the method of moments for proving weak convergence. In the present context, cumulants have two main advantages over moments: first, the cumulants of the Poisson and Gaussian integrals appearing in the limit have a simple structure (see Sect. 1.4); second, cumulants are well adapted to aggregation, since the cumulant of a sum of independent random variables is equal to the sum of cumulants of each term. 


\subsection{Proof of Propositions 1.2 and 1.3}

We first consider the Proof of Proposition 1.2. According to [11], Lemma 12.13, the Poisson integral

$$
\tilde{N}[g]=\int_{\mathbb{R} \times[0,+\infty)} g(x, u) \tilde{N}(\mathrm{~d} x, \mathrm{~d} u)
$$

exists for any measurable function $g: \mathbb{R} \times[0,+\infty) \rightarrow \mathbb{R}$ such that

$$
\int_{\mathbb{R} \times[0,+\infty)}|g(x, u)| \wedge g(x, u)^{2} n(\mathrm{~d} x, \mathrm{~d} u)<\infty .
$$

For $k \geq 1$, the $k$ th moment $\mathbb{E}\left[\tilde{N}[g]^{k}\right]$ and $k$ th cumulant $C_{k}(\tilde{N}[g])$ of $\tilde{N}[g]$ are well-defined as soon as $\int_{\mathbb{R} \times[0,+\infty)}|g(x, u)|^{k} n(\mathrm{~d} x, \mathrm{~d} u)<\infty$. Then, $C_{1}(\tilde{N}[g])=0$ and for $k \geq 2$,

$$
C_{k}(\widetilde{N}[g])=\int_{\mathbb{R} \times[0,+\infty)} g(x, u)^{k} n(\mathrm{~d} x, \mathrm{~d} u) .
$$

Corresponding remarks apply to stochastic integrals with respect to $\widetilde{N}_{+}(\mathrm{d} x, \mathrm{~d} u)$. Therefore, in order to prove that the Poisson integrals $J_{\beta}^{\text {eq }}[f]$ and $J_{\beta}^{0}[f]$ exists and have finite cumulants of all order, it is enough to show that, for all $k \geq 1$,

$$
\int_{\mathbb{R} \times[0,+\infty)}|g(x, u)|^{k} n(\mathrm{~d} x, \mathrm{~d} u)<\infty \quad \text { and } \quad \int_{\mathbb{R} \times[0,+\infty)}|g(x, u)|^{k} n_{+}(\mathrm{d} x, \mathrm{~d} u)<\infty
$$

with $g(x, u)=\int_{[0,+\infty)} 1_{[x, x+u]}(y) f(y) \mathrm{d} y$. This follows easily from the fact that $f$ is bounded and has bounded support. Then, using equation (3.1), we compute

$$
\begin{aligned}
C_{k}\left(J_{\beta}^{\mathrm{eq}}[f]\right) & =\int_{\mathbb{R} \times[0,+\infty)}\left(\int_{[0,+\infty)} 1_{[x, x+u]}(y) f(y) \mathrm{d} y\right)^{k} n(\mathrm{~d} x, \mathrm{~d} u) \\
& =\int_{\mathbb{R} \times[0,+\infty)} \mathrm{d} x(\beta+1) u^{-\beta-2} \mathrm{~d} u \int_{[0,+\infty)^{k}} 1_{y_{(k)}-u \leq x \leq y_{(1)}} f\left(y_{1}\right) \ldots f\left(y_{k}\right) \mathrm{d} y_{1} \ldots \mathrm{d} y_{k} \\
& =\frac{1}{\beta} \int_{[0,+\infty)^{k}} f\left(y_{1}\right) \ldots f\left(y_{k}\right)\left|y_{(k)}-y_{(1)}\right|^{-\beta} \mathrm{d} y_{1} \ldots \mathrm{d} y_{k}=\frac{1}{\beta} \widetilde{P}_{k}^{\mathrm{eq}}\left[f^{\otimes k}\right] .
\end{aligned}
$$

By restricting the $\mathrm{d} x$-integration to the positive half axis rather than the real line as above, we obtain

$$
\begin{aligned}
C_{k}\left(J_{\beta}^{0}[f]\right) & =\int_{[0,+\infty)^{2}}\left(\int_{[0,+\infty)} 1_{[x, x+u]}(y) f(y) \mathrm{d} y\right)^{k}(\beta+1) u^{-\beta-2} \mathrm{~d} u \mathrm{~d} x \\
& =\frac{1}{\beta} \int_{[0,+\infty)^{k}} f\left(y_{1}\right) \ldots f\left(y_{k}\right)\left(\left|y_{(k)}-y_{(1)}\right|^{-\beta}-\left|y_{(k)}\right|^{-\beta}\right) \mathrm{d} y_{1} \ldots \mathrm{d} y_{k}=\frac{1}{\beta} \widetilde{P}_{k}^{0}\left[f^{\otimes k}\right] .
\end{aligned}
$$

It remains to prove that the distributions of $J_{\beta}^{\text {eq }}[f]$ and $J_{\beta}^{0}[f]$ are uniquely determined by their sequence of cumulants. To this aim, we show that the cumulant generating function

$$
\log \mathbb{E} \mathrm{e}^{\theta J_{\beta}^{\mathrm{eq}}[f]}=\sum_{k=1}^{\infty} \frac{\theta^{k}}{k !} C_{k}\left(J_{\beta}^{\mathrm{eq}}[f]\right)
$$

has a positive radius of convergence and similarly for $J_{\beta}^{0}[f]$. Indeed, there exist $M>0$ and $T>0$ such that $|f(y)| \leq M 1_{[0, T]}(y)$ for all $y \in[0,+\infty)$, and then

$$
\left|C_{k}\left(J_{\beta}^{0}\left[f_{c}\right]\right)\right| \leq M^{k}\left|C_{k}\left(J_{\beta}^{0}\left[1_{[0, T]}\right]\right)\right| \leq M^{k}\left|C_{k}\left(J_{\beta}^{\mathrm{eq}}\left[1_{[0, T]}\right]\right)\right|=\frac{M^{k} T^{k-\beta} k(k-1)}{\beta(k+1-\beta)(k+2-\beta)} .
$$

The claim follows. 
The Proof of Proposition 1.3 is easier and based on the same arguments. The Gaussian integral

$$
W[g]=\int_{\mathbb{R} \times[0,+\infty)} g(x, u) \widetilde{W}(\mathrm{~d} x, \mathrm{~d} u)
$$

is well defined for $g \in L^{2}(\mathbb{R} \times[0,+\infty), n)$ and then, $W[g]$ is a centered Gaussian variable with variance

$$
C_{2}(W[g])=\int_{\mathbb{R} \times[0,+\infty)} g(x, u)^{2} n(\mathrm{~d} x, \mathrm{~d} u) .
$$

The remaining cumulants $C_{k}(W[g]), k=1$, or $k \geq 3$, are equal to 0 . Corresponding remarks hold for stochastic integrals with respect to $W_{+}$. Further details are omitted.

\subsection{Proof of Theorem 1.4}

We begin the proof by restating the convergence results for centered moments in Theorem 1.1 in a form which is adapted to the scaling assumptions (ICR) and (FCR). Recall that $\beta$ and $\ell$ are given in (1.2) and that, in the scaling (ICR), the sequence $a=a_{m}$ satisfies $a \rightarrow \infty$ and $m \ell(a) / a^{\beta} \rightarrow \mu c^{\beta}$ as $m \rightarrow \infty$. With $b(a, m)=a$, Theorem 1.1 implies, for $k \geq 1$,

$$
\frac{m}{b^{k}} \widetilde{M}_{k}^{\pi} s_{a}^{-1}=\frac{m \ell(a)}{a^{\beta}} \frac{1}{a^{k-\beta} \ell(a)} \widetilde{M}_{k}^{\pi} s_{a}^{-1} \stackrel{\mathcal{F}_{k}}{\longrightarrow}(-1)^{k} \frac{c^{\beta}}{\beta \mu^{k}} \widetilde{P}_{k}^{\pi} .
$$

Turning to fast connection rate scaling (FCR), we have $m \ell(a) / a^{\beta} \rightarrow \infty$ and norming sequence $b(a, m)=$ $\left(m a^{2-\beta} \ell(a)\right)^{1 / 2}$. Hence, for $k=2$

$$
\frac{m}{b^{2}} \widetilde{M}_{2}^{\pi} s_{a}^{-1}=\frac{1}{a^{2-\beta} \ell(a)} \widetilde{M}_{2}^{\pi} s_{a}^{-1} \stackrel{\mathcal{F}_{2}}{\longrightarrow} \frac{1}{\beta \mu^{3}} \widetilde{P}_{2}^{\pi}
$$

and, for $k \geq 3$,

$$
\begin{aligned}
\frac{m}{b^{k}} \widetilde{M}_{k}^{\pi} s_{a}^{-1} & =\left(\frac{a^{\beta}}{m \ell(a)}\right)^{k / 2-1} \frac{1}{a^{k-\beta} \ell(a)} \widetilde{M}_{k}^{\pi} s_{a}^{-1} \sim\left(\frac{a^{\beta}}{m \ell(a)}\right)^{k / 2-1} \frac{(-1)^{k}}{\beta \mu^{k+1}} \widetilde{P}_{k}^{\pi} \\
& \stackrel{\mathcal{F}_{k}}{\longrightarrow} 0 .
\end{aligned}
$$

Based on the above relations we are able to derive the asymptotic behavior of the cumulants of $\widetilde{\xi}_{a, m}[f]$. As in Section 1.4 we write $C_{k}(X)$ for the $k$ th order cumulant of a random variable $X$, so that $\log \mathbb{E} \mathrm{e}^{\theta X}=\sum_{k=1}^{\infty} \frac{\theta^{k}}{k !} C_{k}(X)$. It is convenient to write $C_{k}^{\pi}(X)$ to emphasize that expectations over $X$ are taken with respect to $\mathbb{E}_{\pi}$ with $\pi=\delta_{0}$ or $\pi=$ eq, and $C_{k}^{0}(X)$ and $C_{k}^{\mathrm{eq}}(X)$ if there is a need to distinguish the two cases.

Lemma 3.1. Under both scaling assumptions (ICR) and (FCR), with the corresponding choice of norming sequence $b(a, m)$, for any $f \in \mathcal{F}_{1}$ we have $C_{1}^{\pi}\left(\widetilde{\xi}_{a, m}[f]\right)=0$ and, for $k \geq 2$,

$$
C_{k}^{\pi}\left(\widetilde{\xi}_{a, m}[f]\right)=\frac{m}{b^{k}}\left(\widetilde{M}_{k}^{\pi} s_{a}^{-1}\right)\left[f^{\otimes k}\right]+o(1) \quad \text { as } m \rightarrow+\infty .
$$

Proof. Since the centering of $\widetilde{\xi}_{a, m}[f]$ is prescribed with respect to the relevant measure $\mathbb{E}_{\pi}$, and hence

$$
\ln \mathbb{E}_{\pi} \mathrm{e}^{\theta \widetilde{\xi}_{a, m}[f]}=m \ln \mathbb{E}_{\pi} \mathrm{e}^{\theta \xi s_{a}^{-1} / b}-\frac{m}{b} \theta \mathbb{E}_{\pi} \xi s_{a}^{-1},
$$

we have

$$
C_{1}^{\pi}\left(\widetilde{\xi}_{a, m}[f]\right)=0
$$


and

$$
C_{k}^{\pi}\left(\widetilde{\xi}_{a, m}[f]\right)=\frac{m}{b^{k}} C_{k}^{\pi}\left(\xi s_{a}^{-1}[f]\right), \quad k \geq 2 .
$$

Moreover, for $k \geq 2$ the cumulants of $\xi s_{a}^{-1}[f]$ and of $\left(\xi s_{a}^{-1}-(a / \mu) \lambda\right)[f]$ coincide. Thus, by applying the general recursive relation between moments and cumulants given by

$$
C_{k}(X)=\mathbb{E}\left[X^{k}\right]-\sum_{i=1}^{k-1}\left(\begin{array}{c}
k-1 \\
i-1
\end{array}\right) C_{i}(X) \mathbb{E}\left[X^{k-i}\right]
$$

to $X=\left(\xi s_{a}^{-1}-(a / \mu) \lambda\right)[f]$, we obtain, for $k \geq 2$,

$$
C_{k}^{\pi}\left(\left(\xi s_{a}^{-1}\right)[f]\right)=\left(\widetilde{M}_{k}^{\pi} s_{a}^{-1}\right)\left[f^{\otimes k}\right]-\sum_{i=1}^{k-1}\left(\begin{array}{c}
k-1 \\
i-1
\end{array}\right) C_{i}^{\pi}\left(\left(\xi s_{a}^{-1}\right)[f]\right)\left(\widetilde{M}_{k-i}^{\pi} s_{a}^{-1}\right)\left[f^{\otimes k-i}\right] .
$$

Hence,

$$
C_{k}^{\pi}\left(\widetilde{\xi}_{a, m}[f]\right)=\frac{m}{b^{k}}\left(\widetilde{M}_{k}^{\pi} s_{a}^{-1}\right)\left[f^{\otimes k}\right]-R_{k}[f]
$$

where

$$
R_{k}[f]=\sum_{i=1}^{k-1}\left(\begin{array}{c}
k-1 \\
i-1
\end{array}\right) C_{i}^{\pi}\left(\widetilde{\xi}_{a, m}[f]\right) \frac{\left(\widetilde{M}_{k-i}^{\pi} s_{a}^{-1}\right)\left[f^{\otimes k-i}\right]}{b^{k-i}} .
$$

It remains to prove that asymptotically $R_{k}[f]=o(1)$. For a proof by induction on $k$, we notice $R_{2}[f]=0$ and suppose that

$$
C_{i}^{\pi}\left(\widetilde{\xi}_{a, m}[f]\right)=\frac{m}{b^{i}}\left(\widetilde{M}_{i}^{\pi} s_{a}^{-1}\right)\left[f^{\otimes i}\right]+o(1), \quad 2 \leq i \leq k-1 .
$$

Here, $f^{\otimes i} \in \mathcal{F}_{i}$ since $f \in \mathcal{F}_{1}$. In view of (3.2) for (ICR) scaling and (3.3), (3.4) for (FCR) we have

$$
\frac{m}{b^{i}}\left(\widetilde{M}_{i}^{\pi} s_{a}^{-1}\right)\left[f^{\otimes i}\right]=O(1)
$$

from which then follows $R_{k}[f]=O(1 / m)$, and hence (3.5) is true for $i=k$.

Proof of Theorem 1.4. We consider the scaling regime (ICR). Minor modifications yield the case (FCR).

For $k \geq 2, f^{\otimes k} \in \mathcal{F}_{k}$. Hence, by Lemma 3.1 and (3.2),

$$
C_{k}^{\pi}\left(\widetilde{\xi}_{a, m}[f]\right) \rightarrow(-1)^{k} \frac{c^{\beta}}{\beta \mu^{k}} \widetilde{P}_{k}^{\pi}\left[f^{\otimes k}\right] .
$$

The scaling property

$$
P_{k}^{\pi}\left[f_{c}^{\otimes k}\right]=c^{\beta} P_{k}\left[f^{\otimes k}\right], \quad \widetilde{P}_{k}^{\pi}\left[f_{c}^{\otimes k}\right]=c^{\beta} \widetilde{P}_{k}^{\pi}\left[f^{\otimes k}\right]
$$

and the property of Poisson integrals (1.8) imply that the limit is

$$
(-1)^{k} \frac{c^{\beta}}{\beta \mu^{k}} \widetilde{P}_{k}^{\pi}\left[f^{\otimes k}\right]=\frac{(-1)^{k}}{\beta \mu^{k}} \widetilde{P}_{k}^{\pi}\left[f_{c}^{\otimes k}\right]=C_{k}\left(-\frac{1}{\mu} J_{\beta}^{\pi}\left[f_{c}\right]\right) .
$$

For $k=1$, the first cumulant of the Poisson integrals vanish, and $C_{1}^{\pi}\left(\widetilde{\xi}_{a, m}[f]\right)=0$ by Lemma 3.1. So, for each $k \geq 1$,

$$
C_{k}^{\pi}\left(\widetilde{\xi}_{a, m}[f]\right) \rightarrow C_{k}\left(-\frac{1}{\mu} J_{\beta}^{\pi}\left[f_{c}\right]\right) .
$$

According to Proposition 1.2, the sequence of cumulants $\left\{C_{k}\left(J_{\beta}^{\pi}\left[-f_{c} / \mu\right]\right), k \geq 1\right\}$ uniquely determines the distribution of $J_{\beta}^{\pi}\left[-f_{c} / \mu\right]$. Hence, by the method of cumulants, we conclude the weak convergence

$$
\widetilde{\xi}_{a, m}[f] \Longrightarrow-\frac{1}{\mu} J_{\beta}^{\pi}\left[f_{c}\right]
$$




\section{A LÉvy PROCESS RENEWAL MODEL}

We are now going to replace the renewal sequence $\left(S_{n}\right)$ with a Lévy subordinator $\left(Z_{s}\right)$, so that the role of the renewal counting process $\xi([0, x])$ is taken over by the first passage times of the Lévy process

$$
\eta([0, x])=\int_{0}^{\infty} 1_{\left\{0 \leq Z_{s} \leq x\right\}} \mathrm{d} s, \quad x \geq 0 .
$$

This provides a continuous counterpart of the discrete renewal model. As we will see, the discrete and continuous models share the same structure of moment measures and all results derived for the discrete model can be transfered to the continuous framework. The continuous model is introduced in [9] and studied here in a more general setting.

\subsection{Model and results}

Let $(Z(t))_{t \geq 0}$ be a Lévy subordinator with right-continuous paths, drift zero and Lévy measure $\nu$ on $[0,+\infty)$ with no atom at zero, such that

$$
\int_{0}^{\infty}(1 \wedge x) \nu(\mathrm{d} x)<\infty \text { and } \quad \mu=\int_{0}^{\infty} y \nu(\mathrm{d} y)<\infty
$$

For the initial distribution $\pi$ of $Z(0)$ we will again consider two variations in analogy with the discrete renewal model. The pure subordinator with $Z(0)=0$ is the basic case $\pi=\delta_{0}$. For the stationary case $\pi=\pi_{\text {eq }}$ we put $Z(t)=\widetilde{Z}(t)+Z(0)$, where $\{\widetilde{Z}(t)\}$ is a pure subordinator, $Z(0)$ is a random variable with the equilibrium distribution

$$
\pi_{\mathrm{eq}}([0, x])=\frac{1}{\mu} \int_{0}^{x} \int_{y}^{\infty} \nu(\mathrm{d} u) \mathrm{d} y
$$

and $\{\widetilde{Z}(t)\}$ and $Z(0)$ are independent. As before, the initial distribution is emphasized in the notations $\mathbb{P}_{\pi}$ and $\mathbb{E}_{\pi}$. For the pure Lévy subordinator, we have $\mathbb{E}_{0}(Z(t))=\mu t, t \geq 0$, and

$$
\mathbb{E}_{0}\left[\mathrm{e}^{-\lambda Z(t)}\right]=\mathrm{e}^{-t \Phi(\lambda)}, \quad \Phi(\lambda)=\int_{0}^{\infty}\left(1-\mathrm{e}^{-\lambda y}\right) \nu(\mathrm{d} y), \quad \lambda \geq 0 .
$$

By imposing initially the equilibrium distribution, for which $\mathbb{E}_{\text {eq }}\left[\mathrm{e}^{-\lambda Z(0)}\right]=\frac{\Phi(\lambda)}{\mu \lambda}$, we obtain

$$
\mathbb{E}_{\mathrm{eq}}\left[\mathrm{e}^{-\lambda Z(t)}\right]=\frac{\Phi(\lambda)}{\mu \lambda} \mathrm{e}^{-t \Phi(\lambda)}
$$

We suppose that the Lévy measure $\nu$ has a regularly varying tail with index $1+\beta$ for $\beta \in(0,1)$, i.e.

$$
\int_{x}^{\infty} \nu(\mathrm{d} y) \sim x^{-(1+\beta)} \ell(x), \quad \text { as } x \rightarrow \infty,
$$

for some slowly varying function $\ell$. In addition, we suppose that the lower index $\sigma$ of the subordinator is greater than $\beta$, i.e.

$$
\sigma=\sup \left\{\alpha: \lim _{\lambda \rightarrow \infty} \Phi(\lambda) / \lambda^{\alpha} \rightarrow \infty\right\}>\beta
$$


Let $\eta$ be the occupation measure of the process $Z$, i.e. the random measure on $\left([0,+\infty), \mathcal{B}_{1}\right)$ defined by

$$
\eta(A)=\int_{0}^{\infty} \delta_{Z(s)}(A) \mathrm{d} s, \quad A \in \mathcal{B}_{1}
$$

The Lévy subordinator $Z$ is transient $(Z(t) \rightarrow+\infty$ almost surely as $t \rightarrow+\infty)$, hence $\eta(A)$ is almost surely finite for all bounded sets $A \in \mathcal{B}_{1}$, [1] Chapter 1.4. For measurable, bounded and compactly supported $f:[0,+\infty) \rightarrow$ $\mathbb{R}$, we put

$$
\eta[f]=\int f d \eta=\int_{0}^{\infty} f(Z(s)) \mathrm{d} s
$$

Denote by $L_{k}^{\pi}(A)$ the $k$ th moment measure of $\eta$ given by

$$
L_{k}^{\pi}(A)=\mathbb{E}_{\pi}\left[\eta^{\otimes k}(A)\right], \quad A \in \mathcal{B}_{k} .
$$

Equivalently,

$$
L_{k}^{\pi}(A)=\mathbb{E}_{\pi}\left[\int_{[0,+\infty)^{k}} \delta_{\left(Z\left(s_{1}\right), \ldots, Z\left(s_{k}\right)\right)}(A) \mathrm{d} s_{1} \ldots \mathrm{d} s_{k}\right]
$$

Define also the centered version of the $k$ th moment measure by

$$
\widetilde{L}_{k}^{\pi}(A)=\mathbb{E}_{\pi}\left[\left(\eta-\lambda_{1} / \mu\right)^{\otimes k}(A)\right], \quad A \in \mathcal{B}_{k} \text { bounded }
$$

Interestingly, the asymptotic behavior of the moment measures is the same as for the discrete setting.

Theorem 4.1. Suppose that assumptions (4.2) and (4.3) are satisfied. Then, for $k \geq 1$ and as $a \rightarrow \infty$,

$$
\frac{L_{k}^{\pi} s_{a}^{-1}-(a / \mu)^{k} \lambda_{k}}{a^{k-\beta} \ell(a)} \stackrel{\mathcal{F}_{k}}{\longrightarrow} \frac{1}{\beta \mu^{k+1}} P_{k}^{\pi}, \quad \frac{\widetilde{L}_{k}^{\pi} s_{a}^{-1}}{a^{k-\beta} \ell(a)} \stackrel{\mathcal{F}_{k}}{\longrightarrow} \frac{(-1)^{k}}{\beta \mu^{k+1}} \widetilde{P}_{k}^{\pi} .
$$

As remarked initially in this section, if we take $f$ to be the indicator function of an interval $[0, x]$ then $\eta[f]$ provides the inverse of the Lévy-subordinator $Z$, given by the process of passage times

$$
\eta([0, x])=\inf \{s \geq 0 ; Z(s) \geq x\}, \quad x \geq 0 .
$$

The expected value $L_{1}^{0}([0, x])=\mathbb{E}_{0}[\eta([0, x])]$ is the pure subordinator renewal function. Under $\pi=\pi_{\text {eq }}$, by stationarity, $L_{1}^{\mathrm{eq}}([x, x+y])=\mathbb{E}_{\mathrm{eq}}[\eta([x, x+y])]=y / \mu$, see [1]. Similarly as in Section 1.5 , the result in Theorem 4.1 provides limit theorems in the continuous setting for the superposition of random sources. Let $\eta^{i}, i \geq 1$ be i.i.d. copies of the random measure $\eta$. We consider

$$
\widetilde{\eta}_{a, m}=\frac{1}{b(a, m)} \sum_{i=1}^{m}\left(\eta^{i} s_{a}^{-1}-L_{1}^{\pi} s_{a}^{-1}\right) .
$$


The counterpart of Theorem 1.4 is the following:

\section{Theorem 4.2.}

(1) Under the rescaling assumption (ICR) with normalization $b(a, m)=a$, for the pure case $\pi=\delta_{0}$,

$$
\widetilde{\eta}_{a, m}[f] \stackrel{f i d i}{\longrightarrow}-\frac{1}{\mu} J_{\beta}^{0}\left[f_{c}\right], \quad f \in \mathcal{F}_{1}
$$

and for the stationary case $\pi=\pi_{\mathrm{eq}}$,

$$
\widetilde{\eta}_{a, m}[f] \stackrel{f i d i}{\longrightarrow}-\frac{1}{\mu} J_{\beta}^{\mathrm{eq}}\left[f_{c}\right], \quad f \in \mathcal{F}_{1} .
$$

(2) With scaling (FCR) and normalization $b(a, m)=\left(m a^{2-\beta} \ell(a)\right)^{1 / 2}$, in the pure case $\pi=\delta_{0}$

$$
\widetilde{\eta}_{a, m}[f] \stackrel{\text { fidi }}{\longrightarrow} G_{\beta}^{0}[f], \quad f \in \mathcal{F}_{1}
$$

and in the stationary case $\pi=\pi_{\mathrm{eq}}$

$$
\widetilde{\eta}_{a, m}[f] \stackrel{f i d i}{\longrightarrow} G_{\beta}^{\mathrm{eq}}[f], \quad f \in \mathcal{F}_{1} .
$$

The main result in [9] is a weak convergence result under (ICR) of the form

$$
\frac{1}{a} \sum_{i=1}^{m}\left(\eta^{i}([0, a x])-\frac{1}{\mu} a x\right) \longrightarrow-\frac{1}{\mu} c J_{\beta}^{\mathrm{eq}}[x / c],
$$

and a similar result under (FCR) with the fractional Brownian motion as limit process.

\subsection{Proof of Theorem 4.1}

The discrete renewal measure $U$ is replaced for the continuous model by the (pure) subordinator renewal measure

$$
V(\mathrm{~d} y)=\mathbb{E}_{0}\left[\int_{0}^{\infty} \delta_{Z(s)}(\mathrm{d} y) \mathrm{d} s\right] .
$$

The following lemma replaces Lemmas 2.3 and 2.5. We use the same notations.

Lemma 4.3. For any $k \geq 1$, the $k$ th moment measure $L_{k}^{\pi}$ satisfies

$$
L_{k}^{\pi}(A)=\sum_{\sigma \in \Sigma_{k}}\left[(\pi * V) \otimes V^{\otimes(k-1)}\right] \theta_{k}^{-1} \sigma^{-1}(A) .
$$

Moreover, for $k \geq 2$,

$$
\widetilde{L}_{k}^{\pi}(A)=\sum_{r=1}^{k} \sum_{|I|=r}(-1)^{k-r}\left(L_{r}^{\pi}-\frac{\lambda_{r}}{\mu^{r}}\right)\left(A_{I}\right) \frac{\lambda_{k-r}\left(A_{I^{c}}\right)}{\mu^{k-r}} .
$$

Proof. For the first statement, split the integral expression in the defining relation (4.4) in $k$ ! terms corresponding to the relative order of $s_{i}, 1 \leq i \leq k$, to obtain

$$
\begin{aligned}
L_{k}^{\pi}(A) & =\mathbb{E}_{\pi} \sum_{\sigma \in \Sigma_{k}} \int_{[0,+\infty)^{k}} \delta_{\left(Z\left(s_{1}\right), \ldots, Z\left(s_{k}\right)\right)}(A) 1_{\left\{s_{\sigma(1)}<\ldots<s_{\sigma(k)}\right\}} \mathrm{d} s_{1} \ldots \mathrm{d} s_{k} \\
& =\sum_{\sigma \in \Sigma_{k}} \mathbb{E}_{\pi} \int_{0<s_{1}<\ldots<s_{k}} \delta_{\left(Z\left(s_{1}\right), \ldots, Z\left(s_{k}\right)\right)}\left(\sigma^{-1} A\right) \mathrm{d} s_{1} \ldots \mathrm{d} s_{k} \\
& =\sum_{\sigma \in \Sigma_{k}} \mathbb{E}_{\pi} \int_{0<s_{1}<\ldots<s_{k}} \delta_{\left(Z\left(s_{1}\right), Z\left(s_{2}\right)-Z\left(s_{1}\right), \ldots, Z\left(s_{k}\right)-Z\left(s_{k-1}\right)\right)}\left(\theta_{k}^{-1} \sigma^{-1} A\right) \mathrm{d} s_{1} \ldots \mathrm{d} s_{k} .
\end{aligned}
$$


By the Markov property and the fact that $Z(0), Z\left(s_{1}\right)-Z(0), Z\left(s_{2}\right)-Z\left(s_{1}\right), \ldots, Z\left(s_{k}\right)-Z\left(s_{k-1}\right)$ are independent,

$$
\mathbb{E}_{\pi} \int_{0<s_{1}<\ldots<s_{k}} \delta_{\left(Z\left(s_{1}\right), Z\left(s_{2}\right)-Z\left(s_{1}\right), \ldots, Z\left(s_{k}\right)-Z\left(s_{k-1}\right)\right)}\left(\theta_{k}^{-1} \sigma^{-1} A\right) \mathrm{d} s_{1} \ldots \mathrm{d} s_{k}=\left[(\pi * V) \otimes V^{\otimes(k-1)}\right]\left(\theta_{k}^{-1} \sigma^{-1} A\right) .
$$

The proof of the remaining formula is the same as that of Lemma 2.5.

The Proof of Theorem 4.1 follows the same lines as the proof of Lemma 2.4, with Lemma 4.3 replacing Lemmas 2.3 and 2.5. The only part in this line of arguments that needs to be considered further is to establish an analogous result to Teugel's estimate (2.3), valid for the subordinator renewal measure $V$. This is the content of Lemma 4.4 below. The proof relies on fine properties of the Lévy measure $\nu$, taking into account its regularly varying tail function in (4.2), which we denote here by

$$
\bar{\nu}(x)=\nu((x, \infty)),
$$

as well as the lower index assumption in (4.3).

Lemma 4.4. As $x \rightarrow \infty$,

$$
V([0, x])-\frac{x}{\mu} \sim \frac{\ell(x) x^{1-\beta}}{\mu^{2} \beta(1-\beta)} .
$$

Proof. For convenience we denote by $\left(T_{x}\right)_{x \geq 0}$, the inverse Lévy-subordinator $T_{x}=\eta([0, x])$ in $(4.5)$, for which we have $\mathbb{E}_{\text {eq }}\left[T_{x}\right]=x / \mu$ and $\mathbb{E}_{0}\left[T_{x}\right]=V([0, x])$. The renewal theorem, see [1] III.1, implies that

$$
V([0, x]) \sim \frac{x}{\mu} \text { as } x \rightarrow \infty .
$$

Define also

$$
\widetilde{T}_{x}=\inf \{s \geq 0 ; Z(s)-Z(0) \geq x\}=\eta([0, x+Z(0)]), \quad x \geq 0 .
$$

By the Markov property, the distribution of $\widetilde{T}_{x}$ under $\mathbb{P}_{\pi}$ is equal to the distribution of $T_{x}$ under $\mathbb{P}_{0}$, so that $\mathbb{E}_{\mathrm{eq}}\left[\widetilde{T}_{x}\right]=V([0, x])$. Hence, conditioning on $Z(0)$,

$$
\begin{aligned}
V([0, x])-x / \mu & =\mathbb{E}_{\mathrm{eq}}\left[\widetilde{T}_{x}-T_{x}\right] \\
& =\int_{0}^{\infty} \mathbb{E}_{y}\left[\widetilde{T}_{x}-T_{x}\right] \pi^{\mathrm{eq}}(\mathrm{d} y) \\
& =\int_{0}^{x}\left(\mathbb{E}_{0}\left[T_{x}\right]-\mathbb{E}_{0}\left[T_{x-y}\right]\right) \pi^{\mathrm{eq}}(\mathrm{d} y)+\int_{x}^{\infty} \mathbb{E}_{0}\left[T_{x}\right] \pi^{\mathrm{eq}}(\mathrm{d} y) \\
& =\int_{0}^{x} V((x-y, x]) \pi^{\mathrm{eq}}(\mathrm{d} y)+V([0, x]) \pi^{\mathrm{eq}}((x,+\infty]) .
\end{aligned}
$$

The assumptions (4.1) and (4.2), and Karamata's theorem [3], imply

$$
\pi^{\mathrm{eq}}((x,+\infty)) \sim \frac{x^{-\beta} \ell(x)}{\mu \beta}, \quad \text { as } x \rightarrow \infty,
$$

so that

$$
V([0, x])-x / \mu \sim \int_{0}^{x} V((x-y, x]) \pi^{\mathrm{eq}}(\mathrm{d} y)+\frac{x^{1-\beta} \ell(x)}{\mu^{2} \beta}, \quad \text { as } x \rightarrow \infty .
$$

Since $\pi^{\mathrm{eq}}(\mathrm{d} z)=\frac{1}{\mu} \bar{\nu}(z) \mathrm{d} z$ by $(4.1)$,

$$
\begin{aligned}
\int_{0}^{x} V((x-y, x]) \pi^{\mathrm{eq}}(\mathrm{d} y) & =\frac{1}{\mu} \int_{0}^{x} V((x-y, x]) \bar{\nu}(y) \mathrm{d} y \\
& =\frac{x^{1-\beta} \ell(x)}{\mu} \int_{0}^{1} \frac{V(x(1-y, 1])}{x} \frac{\bar{\nu}(x y)}{x^{-(1+\beta)} \ell(x)} \mathrm{d} y .
\end{aligned}
$$


Using regular variation, for all $y \in(0,1)$ as $x \rightarrow \infty$,

$$
\frac{V(x(1-y, 1])}{x} \rightarrow \frac{y}{\mu} \quad \text { and } \quad \frac{\bar{\nu}(x y)}{x^{-(1+\beta)} \ell(x)} \rightarrow y^{-(1+\beta)},
$$

so we expect

$$
\int_{0}^{1} \frac{V(x(1-y, 1])}{x} \frac{\bar{\nu}(x y)}{x^{-(1+\beta)} \ell(x)} \mathrm{d} y \rightarrow \frac{1}{\mu} \int_{0}^{1} y^{-\beta} \mathrm{d} y=\frac{1}{\mu(1-\beta)}, \quad \text { as } x \rightarrow \infty .
$$

The Lemma would then be a direct consequence of (4.7)-(4.9). It remains to justify the convergence (4.9). By Lebesgue's dominated convergence theorem, it is enough to provide a suitable domination condition. To this aim, we will need the following estimate which is proved in Appendix A: for $\delta>0$ there exists $x_{0}>0$ and $C>0$ such that

$$
\sup _{x \geq x_{0}} \frac{\bar{\nu}(x y)}{x^{-(1+\beta)} \ell(x)} \leq C y^{-1-\beta-\delta}, \quad y \in(0,1) .
$$

Furthermore, using the inequalities $\mathbb{E}_{0}\left[T_{y+y^{\prime}}\right] \leq \mathbb{E}_{0}\left[T_{y}\right]+\mathbb{E}_{0}\left[T_{y^{\prime}}\right]$ and $0 \leq \mathbb{E}_{0}\left[T_{y}\right]-y / \mu \leq e / \Phi(1 / y)$ (see $[1]$, Sect. III.1), we obtain

$$
\frac{V(x(1-y, 1])}{x} \leq \frac{V([0, x y])}{x} \leq \frac{y}{\mu}+\frac{e}{x \Phi(1 /(x y))} .
$$

The condition (4.3) on the lower index of the Lévy process ensures that for all $p \in(\beta, \sigma), \Phi(\lambda) / \lambda^{p} \rightarrow \infty$ as $\lambda \rightarrow \infty$. On the other hand, $\Phi(\lambda) \sim \lambda \mu$ as $\lambda \rightarrow 0^{+}$and $\Phi$ is a continuous positive function on $(0,+\infty)$. So for $p \in(\beta, \sigma \wedge 1)$, there exists some $C>0$ such that $\Phi(\lambda) \geq C\left(\lambda \wedge \lambda^{p}\right)$ for all $\lambda \geq 0$. As a consequence, for all $y \in(0,1)$ and $x \geq x_{0}$,

$$
\frac{V(x(1-y, 1])}{x} \leq \frac{y}{\mu}+\frac{e}{C} \max \left(y, y^{p} x_{0}^{p-1}\right) .
$$

This bound together with (4.10) imply that there is $C>0$ such that for all $x \geq x_{0}$ and $y \in(0,1)$

$$
\frac{V(x(1-y, 1])}{x} \frac{\bar{\nu}(x y)}{x^{-(1+\beta)} \ell(x)} \leq C y^{p-1-\beta-\delta} .
$$

Since $p-1-\beta>-1$, choosing $\delta$ small enough provides a suitable domination condition. This proves the convergence (4.9) and hence the lemma.

Note also that equation (2.4) holds with $U$ replaced by $V$ and the proof is the same.

\subsection{Proof Theorem 4.2}

Just as in the discrete case, Theorem 4.1 implies Theorem 4.2 thanks to the method of cumulants. The proof is exactly the same as for Theorem 1.4 and we omit the details.

\section{Appendix A. TeChnichl Results}

We gather in this appendix some technical results and proofs relying on the theory of regularly varying functions, see e.g. [3] for background and details. We recall the so called Potter bounds (see Prop. 0.8.ii) in [16]). If $h:[0,+\infty) \rightarrow \mathbb{R}$ is regularly varying at infinity with index $\alpha \in \mathbb{R}$, then for any $\delta>0$ and $t_{0}>0$, there exists $y_{0}$ such that for $t \geq t_{0}$ and $y \geq y_{0}$,

$$
(1-\delta) t^{\alpha-\delta} \leq \frac{h(y t)}{h(y)} \leq(1+\delta) t^{\alpha+\delta}
$$


Proof of (2.4). Consider the function $y \mapsto h(y)=U([0, y])-y / \mu$. The asymptotic relation (2.3) implies that $h$ is regularly varying at infinity with index $\alpha=1-\beta$ and that equation (2.4) is equivalent to

$$
\sup _{u \in[0, x]} \sup _{a \geq a_{0}} \frac{h(a u)}{h(a)}<\infty .
$$

Let $\delta \in(0,1-\beta)$ and $t_{0}=1 / x$. Using the lower Potter bound (A.1), there exists $y_{0}>0$ such that

$$
(1-\delta) t^{1-\beta-\delta} \leq \frac{h(y t)}{h(y)}, \quad t \geq t_{0}, y \geq y_{0}
$$

With the substitution $t \rightarrow 1 / u$ and $y \rightarrow a u$, we obtain

$$
\frac{h(a u)}{h(a)} \leq \frac{1}{1-\delta} u^{1-\beta-\delta}, \quad u \leq x, a u \geq y_{0} .
$$

Note that $1-\beta-\delta>0$. Furthermore, the function $h$ is cad-lag and has limit $+\infty$ at $\infty$. Therefore, $h$ is bounded on $\left[0, y_{0}\right]$ and $1 / h$ is bounded on $\left[a_{0},+\infty\right]$ for some $a_{0}>0$, so that there exists $C>0$ such that

$$
\frac{h(a u)}{h(a)} \leq C, \quad u \leq x, a \geq a_{0}, a u \leq y_{0}
$$

Together these two estimates give the upper bound (2.4).

Proof of (4.10). Since the function $\bar{\nu}$ is regularly varying at infinity with index $-1-\beta$, so that $\bar{\nu}(x) \sim x^{-1-\beta} \ell(x)$ as $x \rightarrow \infty$, we may restate (4.10) as finding for any $\delta>0, C$ and $x_{0}$ such that

$$
\sup _{x \geq x_{0}} \frac{\bar{\nu}(x z)}{\bar{\nu}(x)} \leq C z^{-1-\beta-\delta}, \quad z \in(0,1)
$$

By the lower Potter bound (A.1) with $0<\delta<1$ and $t_{0}=1$, there exists $y_{0}$ such that for $t \geq 1$ and $y \geq y_{0}$,

$$
(1-\delta) t^{-1-\beta-\delta} \leq \frac{\bar{\nu}(y t)}{\bar{\nu}(y)}
$$

With the substitution $t \rightarrow 1 / z$ and $y \rightarrow x z$, we obtain

$$
\frac{\bar{\nu}(x z)}{\bar{\nu}(x)} \leq \frac{1}{1-\delta} z^{-1-\beta-\delta}, \quad z \leq 1, \quad x z \geq y_{0}
$$

We still need a bound when $x z<y_{0}$. For this we take $C>0$ and $x_{0}>0$ such that for $x \geq x_{0}, \bar{\nu}(x) \geq C_{1} x^{-1-\beta-\delta}$. In addition we have the obvious upper bound $\bar{\nu}(x) \leq \mu / x$ valid for any $x$. Thus,

$$
\frac{\bar{\nu}(x z)}{\bar{\nu}(x)} \leq \frac{\mu}{x z} \frac{1}{C_{1} x^{-1-\beta-\delta}}=\frac{\mu}{C_{1}} \frac{1}{z} x^{\beta+\delta}, \quad x \geq x_{0} .
$$

In particular, considering the case $x<y_{0} / z$,

$$
\frac{\bar{\nu}(x z)}{\bar{\nu}(x)} \leq \frac{\mu y_{0}^{\beta+\delta}}{C_{1}} z^{-1-\beta-\delta}, \quad z \leq 1, \quad x z \leq y_{0}, \quad x \geq x_{0} .
$$

Then, we obtain the desired result with $C=\max \left((1-\delta)^{-1}, \mu y_{0}^{\beta+\delta} / C_{1}\right)$.

Acknowledgements. The authors would like to express sincere thanks to a referee for detailed and constructive comments and proposals and for pointing out an error in the first version of the manuscript. 


\section{REFERENCES}

[1] J. Bertoin, Lévy Processes. Cambridge University Press, Cambridge (1996).

[2] H. Biermé, A. Estrade and I. Kaj, Self-similar random fields and rescaled random balls models. J. Theor. Prob. 23 (2010) $1110-1141$.

[3] N.H. Bingham, C.M. Goldie and J.L. Teugels, Regular Variation. Cambridge University Press, Cambridge (1987).

[4] D.J. Daley and D. Vere-Jones, An introduction to the theory of point processes, in Elementary theory and methods I. Probab. Appl. 2nd edition. Springer-Verlag (2003).

[5] C. Dombry and I. Kaj, The on-off network traffic model under intermediate scaling. Queuing Syst. 69 (2011) 29-44,.

[6] R. Gaigalas. A Poisson bridge between fractional Brownian motion and stable Lévy motion. Stoch. Proc. Appl. 116 (2006) 447-462.

[7] R. Gaigalas and I. Kaj, Convergence of scaled renewal processes and a packet arrival model. Bernoulli 9 (2003) $671-703$.

[8] G. Grimmett, Weak convergence using higher-order cumulants. J. Theor. Prob. 5 (1992) 767-773.

[9] I. Kaj and A. Martin-Löf, Scaling limit results for the sum of many inverse Lévy subordinators. arXiv:1203.6831 [math.PR] (2012).

[10] I. Kaj and M.S. Taqqu, Convergence to fractional Brownian motion and to the Telecom process: the integral representation approach, in: In an Out of Equilibrium 2, Progress Probability, vol. 60 edited by M.E. Vares and V. Sidoravicius. Birkhauser (2008) 383-427.

[11] O. Kallenberg. Foundations of Modern Probability, 2nd edition. Springer-Verlag, New York (2002).

[12] J.B. Lévy and M.S. Taqqu, Renewal reward processes with heavy-tailed interrenewal times and heavy-tailed rewards. Bernoulli 6 (2000) 23-44.

[13] T. Mikosch, S. Resnick, H. Rootzen and A. Stegeman, Is network traffic approximated by stable Lévy motion or fractional Brownian motion. Ann. Appl. Probab. 12 (2002) 23-68.

[14] V. Pipiras and M.S. Taqqu, The limit of a renewal-reward process with heavy-tailed rewards is not a linear fractional stable motion. Bernoulli 6 (2000) 607-614.

[15] V. Pipiras, M.S. Taqqu and J.B. Lévy, Slow, fast and arbitrary growth conditions for renewal reward processes when the renewals and the rewards are heavy-tailed. Bernoulli 10 (2004) 121-163.

[16] S.I. Resnick, Extreme values, regular variation, and point processes. Springer, New York (1987).

[17] S.I. Resnick, Heavy-tail phenomena, Probabilistic and statistical modeling. Springer Series in Operations Research and Financial Engineering. Springer, New York (2007).

[18] G. Samorodnitsky and M. Taqqu, Stable Non-Gaussian Random Processes. Chapman and Hall (1994).

[19] M.S. Taqqu, W. Willinger and R. Sherman, Proof of a fundamental result in self-similar traffic modeling. Comput. Commun. Rev. 27 (1997) 5-23.

[20] J.L. Teugels, Renewal theorems when the first and the second moment is infinite. Ann. Math. Statist. 39 (1968) $1210-1219$. 